\title{
A mixed norm estimate for the X-ray transform
}

Thomas Wolff

Let $G$ be the space of lines in $\mathbb{R}^{3}$, i.e. the 4-dimensional manifold whose elements are all lines in $\mathbb{R}^{3}$. We can coordinatize $G$ in the following way

$$
\ell=\ell(e, x),
$$

where $e \in S^{2} \backslash\{ \pm 1\}$ is the direction of $\ell$ and $x=x_{\ell}$ is the unique point on $\ell$ which is perpendicular to $e$. We will denote the direction $e$ of $\ell$ by $\ell^{*}$.

The distance on $G$ can be defined using the standard distances on the sphere and in $\mathbb{R}^{3}$ and this identification, thus

$$
d(\ell, m)=\left|x_{\ell}-x_{m}\right|+\theta(\ell, m),
$$

where $\theta(\ell, m)=\theta\left(\ell^{*}, m^{*}\right)$ is the unoriented angle $(\in[0, \pi / 2])$ between $\ell$ and $m$. This distance has the following property. Let $T_{\ell}(a)$ be the cylinder of radius $\delta$, axis $\ell$ and length 1 , centered at the point $a \in \ell$, and let $T_{\ell}=T_{\ell}\left(x_{\ell}\right)$ where $x_{\ell}$ is as defined above. Then for $\sigma \geq \delta$,

$$
\theta(\ell, m) \leq \sigma, \text { and } T_{\ell} \cap T_{m} \neq \varnothing \text { imply } d(\ell, m) \leq C_{0} \sigma,
$$

where $C_{0}$ is a suitable numerical constant.

All metric quantities defined on $G$ refer to the distance $d$.

We will be using mixed norms on $G$ defined in the following way: if $F: G \longrightarrow \mathbb{R}$ then

$$
\|F\|_{L_{e}^{q}\left(L_{x}^{r}\right)} \stackrel{\text { def }}{=}\left(\int_{e \in S^{2}}\left(\int_{\left\{x \in \mathbb{R}^{3}: x \perp e\right\}}|F(e, x)|^{r} d x\right)^{q / r} d e\right)^{1 / q},
$$


where the $x$-integral is with respect to two dimensional Lebesgue measure. We remark that the functions we will be considering will generally be supported in the set $\{(e, x) \in G:\|x\| \leq 1\}$.

The X-ray transform is the map from functions on $\mathbb{R}^{3}$ to functions on $G$ defined by

$$
X f(\ell)=\int_{\ell} f
$$

Our purpose is to prove the following estimate

Theorem 1. If $f: \mathbb{R}^{3} \longrightarrow \mathbb{R}$ and the support of $f$ is contained in the unit disc then

$$
\|X f\|_{L_{e}^{q}\left(L_{x}^{r}\right)} \leq C_{\varepsilon}\|f\|_{p, \varepsilon}
$$

for any $\varepsilon>0$. Here \|\|$_{p, \varepsilon}$ is the inhomogeneous Sobolev norm with $\varepsilon$ derivatives in $L^{p}$, and the exponents are as follows

$$
p=\frac{5}{2}, \quad q=\frac{10}{3}, \quad r=10 \text {. }
$$

The following is an equivalent formulation of Theorem 1 which is easier to work with.

Theorem 2. Let $\Omega$ be a subset of $S^{2} \backslash \pm 1$, let $E$ be a subset of the unit disc in $\mathbb{R}^{3}$, and $\lambda>0$. Assume that for each $e \in \Omega$ there are $m$ $\delta$-separated lines $\ell$ with direction $\ell^{*}=e$ such that

$$
\left|T_{\ell} \cap E\right| \geq \lambda\left|T_{\ell}\right|
$$

Then

$$
|E| \geq C_{\varepsilon}^{-1} \delta^{C \varepsilon} \lambda^{5 / 2} m^{1 / 4}|\Omega|^{3 / 4} \delta^{1 / 2}
$$

Of course, a subset $\left\{m_{j}\right\}$ of a metric space $M$ is called $\delta$-separated if $j \neq k$ implies that the distance from $m_{j}$ to $m_{k}$ is at least $\delta$.

Theorems 1 and 2 are refinements of the result in [7] - the result in [7] corresponds to the case $m=1$. The argument in the present context is more subtle than the argument in [7], but the basic strategy is similar. Let $D(a, r)$ be the ball centered at $a$ with radius $r$. The main work is to prove 
Lemma 0. Theorem 2 is true provided we make the following additional hypothesis on the tubes $T_{\ell}$ : for any $a \in \mathbb{R}^{3}$,

$$
\left|T_{\ell} \cap E \cap D\left(a, \delta^{\varepsilon}\right)\right| \leq \lambda\left(\log \frac{1}{\delta}\right)^{-10}\left|T_{\ell}\right|
$$

A version of property (5) was also used in [7]. We could call it the "two ends" condition, since it expresses the fact that $E \cap T_{\ell}$ is not concentrated near one end of $T_{\ell}$.

We now explain briefly how Theorem 1 fits into the literature. There is a "space time" estimate for the X-ray transform, i.e. an estimate from $L^{p}$ to $L^{q}(G)$, which in the three dimensional case says that

$$
\|X f\|_{L_{e}^{4}\left(L_{x}^{4}\right)} \lesssim\|f\|_{2}
$$

After a result of Oberlin and Stein [6] for the Radon transform, this was proved by Drury [3] with a loss of $\varepsilon$ derivatives and then by Christ [2] as stated. The main conjecture on the Kakeya maximal function can be stated as

$$
\|X f\|_{L_{e}^{3}\left(L_{x}^{\infty}\right)} \lesssim\|f\|_{3, \varepsilon}
$$

and if one interpolates between this conjectural result and Drury's, one obtains the conjectural bound

$$
\|X f\|_{L_{e}^{q}\left(L_{x}^{r}\right)} \lesssim\|f\|_{p, \varepsilon}, \quad \varepsilon>0
$$

for any $p \in(2,3)$, where $q=2 p^{\prime}$ and $1 / r=1-3 / q$. Theorem 1 confirms (6) when $p \leq 5 / 2$.

In [2] it is conjectured that (6) should hold as an endpoint result, i.e. without the loss of $\varepsilon$ derivatives. When $p<5 / 2$ it is conceivable that this can be proved by refining the argument below, but we do not attempt that here. Nor do we attempt a generalization of Theorem 1 to higher dimensions; the natural generalization would be (6) in $\mathbb{R}^{n}$ with

$$
p=\frac{n+2}{2}, \quad q=(n-1) p^{\prime} \quad \text { and } \quad \frac{1}{r}=1-\frac{n}{q} .
$$

The plan of the paper is as follows: sections 1 and 2 are preliminaries to the proof of Lemma 0 , Section 3 is the proof of Lemma 0 and Section 4 is the proof of Theorems 2 and 1. 


\section{Preliminaries.}

Some notation and terminology is as follows: the number $\varepsilon$ is kept fixed throughout the proof of Lemma 0 . We also fix $\delta$, although needless to say the values of all constants must be independent of $\delta$. If $\ell$ is a line then the tubes $T_{\ell}(a)$ and $T_{\ell}$ are as defined in the introduction and in particular have cross section radius $\delta$. We will say that tubes $T_{\ell}$ and $T_{m}$ intersect at angle $\tau$ if $T_{\ell} \cap T_{m} \neq \varnothing$ and $\theta(\ell, m)=\tau$. If $E$ is a set then the notation $|E|$ will be used to denote the Lebesgue measure or cardinality of $E$ depending on the context. The characteristic function of $E$ will be denoted by $\chi_{E}$. The disc of radius $r$ centered at $x$ in a metric space is denoted $D(x, r)$; we remark that we use this notation regardless of whether the metric space is $\mathbb{R}^{3}, G, S^{2}$ or something else. Finally we will use a certain normalization of the entropy of a set, which in practice will be a set in $G$ or on the 2 -sphere.

Definition. If $M$ is a metric space and $\sigma>0$ then $\mathcal{E}_{\sigma}(M)=\sigma^{2} \mathcal{N}_{\sigma}(M)$, where $\mathcal{N}_{\sigma}(M)$ is the maximum possible cardinality for a $\sigma$-separated subset of $M$.

In proving Lemma 0 we can assume that our lines intersect the unit ball in $\mathbb{R}^{3}$ and make an angle of less or equal than 1/100 with the vertical direction, say, and will always make these assumptions in order to avoid some notational complications. We also always assume that $\delta$ is sufficiently small.

In several places we will need to use some elementary but not completely obvious facts from solid geometry. We will generally not give the proofs of these facts. However, we want to clarify our terminology. If $\ell, \ell^{\prime} \in G$ are intersecting lines then the plane spanned by $\ell$ and $\ell^{\prime}$ means of course the unique plane containing $\ell$ and $\ell^{\prime}$. In addition, if $\ell \in G$ and $e \in S^{2}$ then the plane spanned by $\ell$ and $e$ is the set $\left\{x \in \mathbb{R}^{3}: x=y+t e\right.$ for some $y \in \ell$ and $\left.t \in \mathbb{R}\right\}$. If $\Pi$ and $\tilde{\Pi}$ are 2-planes, then the angle between $\Pi$ and $\tilde{\Pi}$ is of course the inverse cosine of the dot product between the unit normal vectors to $\Pi$ and $\tilde{\Pi}$, just as the angle between two lines is is the inverse cosine of the dot product of their direction vectors. As an example of the kind of statement we have in mind, we note the following.

Lemma 1.0. Suppose that $\Pi$ is a plane, $\ell$ is a line contained in $\Pi, \ell^{\prime}$ is a line intersecting $\ell$ at a point $a$, and that the angle between $\ell$ and $\ell^{\prime}$ is 
less or equal than $\sigma$ and the angle between $\Pi$ and the plane spanned by $\ell$ and $\ell^{\prime}$ is $\leq \phi$. Then $T_{\ell^{\prime}}(a)$ is contained in the $C(\phi \sigma+\delta)$-neighborhood of $\Pi$, i.e., if $x \in T_{\ell^{\prime}}(a)$ then $\operatorname{dist}(x, \Pi) \leq C(\phi \sigma+\delta)$.

Proof. Choose coordinates so that $a$ is the origin, $\Pi$ is the $x_{1} x_{2}$ plane, and $\ell$ is the $x_{2}$ axis. Then the assumptions mean that if $y \in \ell^{\prime}$, then

$$
\begin{aligned}
\left|y_{1}\right|+\left|y_{3}\right| & \lesssim \sigma\left(\left|y_{1}\right|+\left|y_{2}\right|+\left|y_{3}\right|\right) \\
\left|y_{3}\right| & \lesssim \phi\left(\left|y_{1}\right|+\left|y_{3}\right|\right) .
\end{aligned}
$$

If $x \in T_{\ell^{\prime}}(a)$, then there is a point $y \in \ell^{\prime}$ with $\left|y_{1}\right|+\left|y_{2}\right|+\left|y_{3}\right| \leq C$ and with $|x-y| \leq C \delta$. The above equations then imply $\left|y_{3}\right| \lesssim \sigma \phi$, so $\left|x_{3}\right| \lesssim \sigma \phi+\delta$ as claimed.

One problem in adapting the argument in [7] is as follows: use was made there of the fact (perhaps due to Córdoba) that a family of tubes contained in a $C \delta$-neighborhood of a 2-plane and with $\delta$-separated directions must satisfy an estimate $\sum_{j}\left|T_{j}\right| \approx\left|\cup_{j} T_{j}\right|$ up to $\delta^{\varepsilon}$ factors. Here we will be considering families of lines which are $\delta$-separated in the Grassmannian $G$, but their directions may not be $\delta$-separated. Lemma 1.2 below is an adaptation of the Córdoba argument to this situation; the form of the statement may look peculiar, but it is the one which is most useful for our purposes.

We will be considering various rectangles $R$ relative to an orthonormal basis $e_{1}, e_{2}, e_{3}$ with respective dimensions $100 \times w \times 100 \delta$, where we always assume that $100>w>100 \delta$. Given such a rectangle $R$, we will call $w$ the width of $R$ and will refer to the plane through the center point of $R$ spanned by the $e_{1}$ and $e_{2}$ directions as the 2-plane of $R$ and to the line through the center in the $e_{1}$ direction as the axis of $R$.

We fix a set $E$ and number $\lambda$. If $\mathcal{A}$ is a $\delta$-separated family of lines and if $R$ is a $100 \times w \times 100 \delta$-rectangle then we define the tube density of $R, d_{\mathcal{A}}(R)$, via

$$
d_{\mathcal{A}}(R)=\frac{\left|\left\{\ell \in \mathcal{A}: T_{\ell} \subset R\right\}\right|}{\frac{w}{\delta}}
$$

A plate of width $w$ relative to $\mathcal{A}$ is a $100 \times w \times 100 \delta$-rectangle $R$ with the following property: 
Plate property. Suppose that for each $\ell \in \mathcal{A}$ with $T_{\ell} \subset R$, a subset $Y_{\ell} \subset T_{\ell} \cap E$ is given, satisfying

$$
\left|Y_{\ell}\right| \geq\left(\log \frac{1}{\delta}\right)^{-3} \lambda\left|T_{\ell}\right|
$$

Then

$$
\left|\bigcup_{T_{\ell} \subset R} Y_{\ell}\right| \geq\left(\log \frac{1}{\delta}\right)^{-10} \lambda^{2}|R|
$$

Assuming that $\mathcal{A}$ is $\delta$-separated and the tubes $\left\{T_{\ell}\right\}_{\ell \in \mathcal{A}}$ satisfy (3), we define a quantity $p_{\sigma}(\mathcal{A})$ in the following way

$$
p_{\sigma}(\mathcal{A})=\sup _{R} d_{\mathcal{A}}(R)
$$

where $R$ runs over all plates relative to $\mathcal{A}$ of width $\leq \sigma$. We will frequently use the fact (easy to prove) that $p_{\sigma}$ is monotone under set inclusion,

$$
\mathcal{B} \subset \mathcal{A} \text { implies } p_{\sigma}(\mathcal{B}) \leq p_{\sigma}(\mathcal{A})
$$

Lemma 1.1. Assume that $\mathcal{A}$ is $\delta$-separated and the tubes $\left\{T_{\ell}\right\}_{\ell \in \mathcal{A}}$ satisfy (3). Then

$$
p_{\sigma}(\mathcal{A})=\sup _{R} d_{\mathcal{A}}(R),
$$

where $R$ runs over all $100 \times w \times 100 \delta$ rectangles with $w \leq \sigma$ (not just plates).

\section{Corollary.}

i) $p_{\sigma}(\mathcal{A})$ actually depends only on $\mathcal{A}$ and not on $E$ or $\lambda$.

ii) Let $\bar{\sigma}=\max \left(100 \delta, \delta^{2 \varepsilon} \sigma\right)$. Then $p_{\bar{\sigma}}(\mathcal{A}) \geq \delta^{5 \varepsilon} p_{\sigma}(\mathcal{A})$.

Proof of the Corollary. Part i) is obvious from Lemma 1.1. Part ii) follows since it is easy to see that if $w^{\prime}=\max \left\{\delta^{2 \varepsilon} w, 100 \delta\right\}$ and if $R$ is a $100 \times w \times 100 \delta$ rectangle which contains $M$ tubes $T_{\ell}, \ell \in \mathcal{A}$, then there must be a $100 \times w^{\prime} \times 100 \delta$-subrectangle containing at least $C^{-1} \delta^{4 \varepsilon} M$ of these tubes. 
Proof of Lemma 1.1. Fix a rectangle $P$ with essentially the maximum tube density, i.e., $P$ is a $100 \times w \times 100 \delta$ rectangle with $w \leq \sigma$, and if $R$ is any other such rectangle, then $d_{\mathcal{A}}(R) \leq 2 d_{\mathcal{A}}(P)$. Let $\mathcal{C}(P)$ be the lines $\ell \in \mathcal{A}$ with $T_{\ell} \subset P$.

It suffices to show that $P$ is a plate relative to $\mathcal{A}$. So fix appropriate subsets $Y_{\ell} \subset T_{\ell}$, which from the form of the statement may be assumed to have measure exactly

$$
\frac{\lambda}{\left(\log \frac{1}{\delta}\right)^{3}}\left|T_{\ell}\right|
$$

Let $\tilde{E}=\cup_{\ell \in \mathcal{C}(P)} Y_{\ell}$. Then, by Córdoba's well-known calculation,

$$
\begin{aligned}
\frac{\lambda}{\left(\log \frac{1}{\delta}\right)^{3}}|\mathcal{C}(P)| \delta^{2} & \approx \sum_{\ell \in \mathcal{C}(P)}\left|Y_{\ell}\right| \\
& =\int_{\tilde{E}} \sum_{\ell \in \mathcal{C}(P)} \chi_{Y_{\ell}} \\
& \leq|\tilde{E} \cap P|^{1 / 2}\left\|\sum_{\ell \in \mathcal{C}(P)} \chi_{Y_{\ell}}\right\|_{2} \\
& =|\tilde{E} \cap P|^{1 / 2}\left(\sum_{\ell, m \in \mathcal{C}(P)}\left|Y_{\ell} \cap Y_{m}\right|\right)^{1 / 2}
\end{aligned}
$$

For each $\ell$ and $\tau \geq \delta$, the maximality property of $P$ implies there are $\lesssim$ $(\tau / w)|\mathcal{C}(P)|$ tubes $T_{m}$ with $m \in \mathcal{C}$ which intersect $T_{\ell}$ at angle between $(\tau-\delta) / 2$ and $\tau$. For each such $m,\left|Y_{\ell} \cap Y_{m}\right| \lesssim \tau^{-1} \delta^{3}$. Accordingly (the sum over $\tau$ below runs over dyadic values between $\delta$ and $\sigma$ )

$$
\begin{aligned}
& \lesssim|\tilde{E} \cap P|^{1 / 2}\left(\sum_{\ell \in \mathcal{C}(P)} \sum_{\tau} \frac{|\mathcal{C}(P)| \tau}{w} \frac{\delta^{3}}{\tau}\right)^{1 / 2} \\
& \lesssim|\tilde{E} \cap P|^{1 / 2}\left(\frac{|\mathcal{C}(P)|^{2} \delta^{3} \log \frac{1}{\delta}}{w}\right)^{1 / 2}
\end{aligned}
$$

and now (8) follows by algebra. 
Lemma 1.2. Let $\mathcal{A}$ be a $\delta$-separated subset of $G$ and assume that the tubes $T_{\ell}, \ell \in \mathcal{A}$ are contained in the intersection of a $\sigma$-neighborhood of a line and $a 100 \delta$-neighborhood of a 2-plane, where $\sigma \geq \delta$. Assume that for each $\ell \in \mathcal{A}$ a subset $Y_{\ell} \subset T_{\ell} \cap E$ is given, satisfying

$$
\left|Y_{\ell}\right| \geq\left(\log \frac{1}{\delta}\right)^{-3} \lambda\left|T_{\ell}\right|
$$

Let $\tilde{E}=\cup_{\ell \in \mathcal{A}} Y_{\ell}$. Then, with $p=p_{\sigma}(\mathcal{A})$,

$$
|\tilde{E}| \geq\left(\log \frac{1}{\delta}\right)^{-10} p^{-1} \lambda^{2} \mathcal{E}_{\delta}(\mathcal{A}) .
$$

Proof. This is similar to the proof of Lemma 1.1. By Lemma 1.1 we know that $|\mathcal{C}(R)| \lesssim p w / \delta$ for all $100 \times w \times 100 \delta$ rectangles $R$. So for any fixed $\ell \in \mathcal{A}$ and $\tau$, there are $\lesssim p \tau / \delta$ tubes which intersect $T_{\ell}$ at angle less or equal than $\tau$. Hence

$$
\begin{aligned}
\frac{\lambda}{\left(\log \frac{1}{\delta}\right)^{3}}|\mathcal{A}| \delta^{2} & \lesssim \sum_{\ell \in \mathcal{A}}\left|Y_{\ell}\right| \\
& \leq|\tilde{E}|^{1 / 2}\left(\sum_{\ell m}\left|Y_{\ell} \cap Y_{m}\right|\right)^{1 / 2} \\
& \lesssim|\tilde{E}|^{1 / 2}\left(\sum_{i} \sum_{\tau} \frac{p \tau}{\delta} \frac{\delta^{3}}{\tau}\right)^{1 / 2} \\
& \lesssim|\tilde{E}|^{1 / 2}\left(|\mathcal{A}| p \delta^{2} \log \frac{1}{\delta}\right)^{1 / 2}
\end{aligned}
$$

using the same type of reasoning as before. The result follows.

The rest of this section is of a technical nature - Lemma 1.4 below will allow us to avoid some unpleasant technicalites later on. Similar issues come up elsewhere in the literature and Lemma 1.3 was suggested by some (rather more sophisticated) lemmas of the same type due to Szemeredi and Balog-Szemeredi, see [5, Section 9.3].

Assume that $\mathcal{A}$ is a set, $N$ a number with $|\mathcal{A}| \leq N$. An allowable relation on $\mathcal{A}$ means a pair $\left\{\Pi_{\mathcal{B}}\right\}_{\mathcal{B} \subset \mathcal{A}}, \sim$, where

1) For each $\mathcal{B} \subset \mathcal{A}, \Pi_{B}$ is a collection of subsets of $\mathcal{B}$. Also $\sim$ is a relation between points of $\mathcal{A}$ and subsets of $\mathcal{A}$ which belong to $\cup_{\mathcal{B} \subset \mathcal{A}} \Pi_{\mathcal{B}}$. 
2) If $\mathcal{B}_{1} \subset \mathcal{B}_{2}$ and if $S_{1} \in \Pi_{\mathcal{B}_{1}}$, then there is $S_{2} \in \Pi_{\mathcal{B}_{2}}$ with $S_{1} \subset S_{2}$ such that $x \sim S_{1}$ implies $x \sim S_{2}$.

3) If $x \in \mathcal{B}$ then there is $S \in \Pi_{\mathcal{B}}$ with $x \sim S$.

If $\mathcal{B} \subset \mathcal{A}, S \in \Pi_{\mathcal{B}}$ then we define $n_{\mathcal{B}}(S)=|\{x \in \mathcal{B}: x \sim S\}| ;$ and $q(\mathcal{B})=\max \left\{n_{\mathcal{B}}(S): S \in \Pi_{\mathcal{B}}\right\}$. We note that property 2) guarantees that $q$ is monotone under set inclusion, $\mathcal{B}_{1} \subset \mathcal{B}_{2}$ implies $q\left(\mathcal{B}_{1}\right) \leq q\left(\mathcal{B}_{2}\right)$. Likewise property 3) guarantees that $q(\mathcal{B}) \geq 1$ for all $\mathcal{B} \subset \mathcal{A}$.

Definition. A subset $\mathcal{A}^{\prime} \subset \mathcal{A}$ is good relative to $\sim$ if the following holds: if $\mathcal{B} \subset \mathcal{A}^{\prime}$ with $|\mathcal{B}| \geq(\log N)^{-10}\left|\mathcal{A}^{\prime}\right|$ then there is a subset $\mathcal{C} \subset \mathcal{B}$ with $|\mathcal{C}| \geq|\mathcal{B}| / 2$ such that $x \in \mathcal{C}$ implies there is $S \in \Pi_{\mathcal{B}}$ such that $x \in S$ and $n_{\mathcal{B}}(S) \geq N^{-\varepsilon} q\left(\mathcal{A}^{\prime}\right)$.

In practice, we will work with several allowable relations simultaneously. Suppose then that $\left\{\left\{\Pi_{\mathcal{B}}^{j}\right\}_{\mathcal{B} \subset \mathcal{A}}, \sim_{j}\right\}_{j=1}^{k}$ is a family of allowable relations on a set $\mathcal{A}$ and denote the quantities $n_{\mathcal{B}}(S)$ and $q(\mathcal{B})$ defined using the relation $\sim_{j}$ by $n_{\mathcal{B}}^{j}(S)$ and $q^{j}(\mathcal{B})$. We say that $\mathcal{A}^{\prime} \subset \mathcal{A}$ is good with respect to all of the relations $\sim_{j}$ if the preceding definition is valid for each $j$, with the set $\mathcal{C}$ being independent of $j$. More precisely,

Definition. A subset $\mathcal{A}^{\prime} \subset \mathcal{A}$ is good relative to all of the relations $\sim_{j}$ if the following holds: if $\mathcal{B} \subset \mathcal{A}^{\prime}$ with $|\mathcal{B}| \geq(\log N)^{-10}\left|\mathcal{A}^{\prime}\right|$ then there is a subset $\mathcal{C} \subset \mathcal{B}$ with $|\mathcal{C}| \geq|\mathcal{B}| / 2$ such that $x \in \mathcal{C}$ implies that for each $j$ there is $S \in \Pi_{\mathcal{B}}^{j}$ such that $x \in S$ and $n_{\mathcal{B}}^{j}(S) \geq N^{-\varepsilon} q^{j}\left(\mathcal{A}^{\prime}\right)$.

The point is that a fairly large "good" subset will always exist:

Lemma 1.3. If $\left\{\sim_{j}\right\}_{j=1}^{k}$ is a family of allowable relations on a set $\mathcal{A}$ with $|\mathcal{A}| \leq N$, and if $N$ is large enough depending on $\varepsilon$ and $k$, then there is a subset $\mathcal{A}^{\prime} \subset \mathcal{A}$ with $\left|\mathcal{A}^{\prime}\right| \geq N^{-\varepsilon}|\mathcal{A}|$ which is good relative to all of the relations $\sim_{j}$.

Proof. Consider a subset of $\mathcal{A}$, which we denote by $\mathcal{A}_{i}$, which is not good with respect to all of the relations $\sim_{j}$. Then, from the definition, there is a subset $\mathcal{B} \subset \mathcal{A}_{i}$ with $|\mathcal{B}| \geq(\log N)^{-10}\left|\mathcal{A}_{i}\right|$, such that half of the elements $x \in \mathcal{B}$ satisfy $\max \left\{n_{\mathcal{B}}^{j}(S): x \in S, S \in \Pi_{\mathcal{B}}^{j}\right\} \leq N^{-\varepsilon} q^{j}\left(\mathcal{A}_{i}\right)$ for some $j$ (depending on $x$ ). Hence we can find a common value of $j$ which works for at least $|\mathcal{B}| /(2 k)$ elements. Defining $\mathcal{A}_{i+1}$ to be these elements, we see that $n_{\mathcal{B}}^{j}(S) \leq N^{-\varepsilon} q^{j}\left(\mathcal{A}_{i}\right)$ for all $S \in \Pi_{\mathcal{B}}^{j}$ such that 
$S \cap \mathcal{A}_{i+1} \neq \varnothing$. Consequently, if $S \in \Pi_{\mathcal{A}_{i+1}}^{j}$ then $n_{\mathcal{A}_{i+1}}^{j}(S) \leq N^{-\varepsilon} q^{j}\left(\mathcal{A}_{i}\right)$ by property 2), and therefore $q^{j}\left(\mathcal{A}_{i+1}\right) \leq N^{-\varepsilon} q^{j}\left(\mathcal{A}_{i}\right)$. We conclude.

If $\mathcal{A}_{i}$ is not good, then there are $\mathcal{A}_{i+1} \subset \mathcal{A}_{i}$ with $\left|\mathcal{A}_{i+1}\right| \geq$ $(\log N)^{-10}\left|\mathcal{A}_{i}\right| /(2 k)$ and $j \in\{1, \ldots, k\}$ such that

$$
q^{j}\left(\mathcal{A}_{i+1}\right) \leq N^{-\varepsilon} q^{j}\left(\mathcal{A}_{i}\right) .
$$

Now suppose we have a string

$$
\mathcal{A}=\mathcal{A}_{0} \supset \cdots \supset \mathcal{A}_{n}
$$

so that the above property holds for each $i=0, \ldots, n-1$. We can pigeonhole to obtain a common value of $j$ for at least $n / k$ values of $i$. Using the monotonicity property of $q^{j}$ it then follows that

$$
1 \leq q^{j}\left(\mathcal{A}_{n}\right) \leq N^{-\varepsilon n / k} q^{j}(\mathcal{A}) \leq N^{-\varepsilon n / k+1},
$$

i.e. $n \leq k / \varepsilon$. On the other hand the last element of a maximal string (13) must be good. So we have found a good subset with at least $\left((\log N)^{-10} /(2 k)\right)^{k / \varepsilon}|\mathcal{A}|$ elements, which gives the result.

We now specialize to the situation we care about, namely the following situation:

(*) $\mathcal{A}$ is a $\delta$-separated subset of $G$ and the tubes $\left\{T_{\ell}\right\}_{\ell \in \mathcal{A}}$ satisfy (3) with respect to some set $E$ contained in the unit ball (and some $\lambda$ ).

If $\mathcal{B} \subset \mathcal{A}$ then we let $P_{j}(\mathcal{B})$ be the set of all plates relative to $\mathcal{B}$ of width less or equal than $\delta^{j \varepsilon}$. If $\ell$ is a line, then we let $P_{j}(\mathcal{B}, \ell)$ be the set of all plates relative to $\mathcal{B}$ of width less or equal than $\delta^{j \varepsilon}$ which contain $T_{\ell}$. Finally, if $R$ is a plate relative to $\mathcal{B}$ then we let $\mathcal{B}_{i r}(R)$ be the set of lines in $\mathcal{B}$ such that the following conditions hold: i) $T_{\ell}$ intersects $R$; and if we denote the axis direction of $R$ by $e$, then ii) the angle between the direction of $\ell$ and the direction of $e$ is less or equal than $\delta^{i \varepsilon}$, and iii) the angle between the 2-plane of $R$ and the 2-plane spanned by $\ell$ and the $e$ direction is less or equal than $\delta^{r \varepsilon}$.

Definition. Suppose that $\mathcal{A}^{\prime} \subset \mathcal{A}$. Then $\mathcal{A}^{\prime}$ is good if for any $\mathcal{B} \subset \mathcal{A}^{\prime}$ with

$$
|\mathcal{B}| \geq\left(\log \frac{1}{\delta}\right)^{-10}\left|\mathcal{A}^{\prime}\right|
$$


there is $\mathcal{C} \subset \mathcal{B}$ with $|\mathcal{C}| \geq|\mathcal{B}| / 2$ such that if $\ell_{0} \in \mathcal{C}$ then

1) For any integer $j \leq 1 / \varepsilon$, we have

$$
\begin{aligned}
\mid\left\{m \in \mathcal{B}: T_{m}\right. & \left.\cap T_{\ell_{0}} \neq \varnothing \text { and } \theta\left(\ell_{0}, m\right) \leq \delta^{j \varepsilon}\right\} \mid \\
& \geq \delta^{\varepsilon} \mid\left\{m \in \mathcal{A}^{\prime}: T_{m} \cap T_{\ell_{0}} \neq \varnothing \text { and } \theta\left(\ell_{0}, m\right) \leq \delta^{j \varepsilon}\right\} \mid .
\end{aligned}
$$

2) For any integer $j$ with $\delta^{j \varepsilon} \geq 100 \delta$, we have

$$
\max _{R \in P_{j}\left(\mathcal{B}, \ell_{0}\right)} d_{\mathcal{B}}(R) \geq \delta^{2 \varepsilon} p_{\sigma}\left(\mathcal{A}^{\prime}\right)
$$

Here we have set $\sigma=\delta^{j \varepsilon}$, and the notation $d_{\mathcal{B}}(R)$ and $p_{\sigma}\left(\mathcal{A}^{\prime}\right)$ is defined by (7) and (9).

3) For any $j$ with $\delta^{j \varepsilon} \geq 100 \delta$ and any $i \leq 1 / \varepsilon, r \leq 1 / \varepsilon$, we have

$$
\max _{R \in P_{j}\left(\mathcal{B}, \ell_{0}\right)}\left|\mathcal{B}_{i r}(R)\right| \geq \delta^{\varepsilon} \max _{R \in P_{j}\left(\mathcal{A}^{\prime}, \ell_{0}\right)}\left|\mathcal{A}_{i r}^{\prime}(R)\right|
$$

Lemma 1.4. If $\mathcal{A}$ is as described by $(*)$ then $\mathcal{A}$ has a good subset $\mathcal{A}^{\prime}$ with $\left|\mathcal{A}^{\prime}\right| \geq \delta^{\varepsilon}|\mathcal{A}|$.

Proof. We will define a set of allowable relations and apply Lemma 1.3. Let $\mathcal{A}$ be our set of lines, $N=\delta^{-4}$ which is clearly an upper bound for $|\mathcal{A}|$. If $\mathcal{B} \subset \mathcal{A}$, and if $R$ is a plate relative to $\mathcal{B}$, then we define a subset $S_{\mathcal{B}}(R)=\left\{m \in \mathcal{B}: T_{m} \subset R\right\}$. We could call this the combinatorial plate corresponding to the geometric plate $R$. We let $\Pi_{j}(\mathcal{B})$ be the set of all "combinatorial plates" relative to $\mathcal{B}$ with width less or equal than $\delta^{j \varepsilon}$, i.e.

$$
\Pi_{j}(\mathcal{B})=\left\{S_{\mathcal{B}}(R): R \in P_{j}(\mathcal{B})\right\}
$$

The following then constitute a set of less or equal than $\varepsilon^{-4}$ allowable relations:

1) ${ }_{j}$ For each $\mathcal{B} \subset \mathcal{A}, \Pi_{\mathcal{B}}$ is all singleton subsets $\{m\}, m \in \mathcal{B}$, with the relations $\ell \sim\{m\}$ if $\theta(\ell, m) \leq \delta^{j \varepsilon}$ and $T_{\ell} \cap T_{m} \neq \varnothing$.

2) ${ }_{j} \Pi_{\mathcal{B}}=\Pi_{j}(\mathcal{B})$ is defined by (16), and $\ell \sim S_{\mathcal{B}}(R)$ if $T_{\ell} \subset R$.

$3)_{i j r} \Pi_{\mathcal{B}}=\Pi_{j}(\mathcal{B})$ is defined by $(16)$; and $\ell \sim S_{\mathcal{B}}(R)$ if $T_{\ell}$ intersects $R, \ell$ makes an angle less or equal than $\delta^{i \varepsilon}$ with the axis direction of 
$R$, and the 2-plane spanned by $\ell$ and the axis direction of $R$ makes an angle less or equal than $\delta^{r \varepsilon}$ with the 2-plane of $R$.

It is almost immediate that all these relations are allowable. We indicate the proof.

Property 2) holds for the relations 1): if $S_{1}=\{m\}$, then take $S_{2}=\{m\}$ also.

Property 2) holds for the relations 2) and 3): if $S_{1} \in \Pi_{j}\left(\mathcal{B}_{1}\right)$ then $S_{1}$ is the combinatorial plate $S_{\mathcal{B}_{1}}(R)$ corresponding to some plate $R \in$ $P_{j}\left(\mathcal{B}_{1}\right)$. Then clearly $R \in P_{j}\left(\mathcal{B}_{2}\right)$ also, and it follows that we can take $S_{2}=S_{\mathcal{B}_{2}}(R)$.

Property 3) holds for the relations 1 ): if $\ell \in \mathcal{B}$ then we can take $S=\{\ell\}$.

Property 3) holds for the relations 2) and 3): for this, fix a line $\ell \in \mathcal{B}$ and set $S=S_{\mathcal{B}}(R)$ where $R$ is a $100 \times w \times 100 \delta$ rectangle containing $T_{\ell}$, with axis very close to and coplanar with $\ell$, and width slightly greater than $100 \delta$. $R$ will be a plate with respect to $\mathcal{B}$ according to our definition and clearly $\ell \sim S$ for any of the relations 2) or 3).

By Lemma 1.3, there is a subset $\mathcal{A}^{\prime} \subset \mathcal{A}$ which is good with respect to all of these relations and has cardinality $\geq \delta^{\varepsilon}|\mathcal{A}|$. Let us now see that this means $\mathcal{A}^{\prime}$ is good in the sense of the preceding definition. Fix an appropriate subset $\mathcal{B}$ and choose a further subset $\mathcal{C}$ using the fact that $\mathcal{A}^{\prime}$ is good with respect to the relations 1), 2), 3). If $\ell_{0} \in \mathcal{C}$ then properties 1) and 3) in the definition of good follow immediately using the relations 1 ) and 3 ). For example, the relation 3$)_{i j r}$ leads to the conclusion

$$
\max _{R \in P_{j}\left(\mathcal{B}, \ell_{0}\right)}\left|\mathcal{B}_{i r}(R)\right| \geq \delta^{\varepsilon} \max _{R \in P_{j}\left(\mathcal{A}^{\prime}\right)}\left|\mathcal{A}_{i r}^{\prime}(R)\right|
$$

which is slightly stronger than (15), and similarly 1$)_{j}$ leads to a slightly stronger form of property 1). It remains to prove (14). The relations $2)_{j}$ imply in the notation (7), (9) that

$$
\max _{R \in P_{k}\left(\mathcal{B}, \ell_{0}\right)} w(R) d_{\mathcal{B}}(R) \geq \delta^{\varepsilon} \max _{R^{\prime} \in P_{k}\left(\mathcal{A}^{\prime}\right)} w\left(R^{\prime}\right) d_{\mathcal{A}^{\prime}}\left(R^{\prime}\right)
$$

for any $k$, where $w(R)$ is the width of $R$. Now let $j$ and $\sigma$ be as in (14) and choose a plate achieving $p_{\sigma}\left(\mathcal{A}^{\prime}\right)$, i.e. let $R^{\prime}$ be a plate relative to $\mathcal{A}^{\prime}$ with width $w^{\prime} \leq \sigma$ and with $p_{\sigma}\left(\mathcal{A}^{\prime}\right)=d_{\mathcal{A}^{\prime}}\left(R^{\prime}\right)$. Choose $k$ as large as possible subject to $\delta^{k \varepsilon} \geq w^{\prime}$, and apply (17). Thus $p_{\sigma}\left(\mathcal{A}^{\prime}\right) \leq$ $\left(\delta^{\varepsilon} w^{\prime}\right)^{-1} \max _{R \in P_{k}\left(\mathcal{B}, \ell_{0}\right)} w(R) d_{\mathcal{B}}(R)$. Now note that $\delta^{k \varepsilon} \leq \delta^{-\varepsilon} w^{\prime}$; we 
conclude therefore that $p_{\sigma}\left(\mathcal{A}^{\prime}\right) \leq \delta^{-2 \varepsilon} \max _{R \in P_{k}\left(\mathcal{B}, \ell_{0}\right)} d_{\mathcal{B}}(R)$. Clearly $k \geq j$, so $P_{k}\left(\mathcal{B}, \ell_{0}\right) \subset P_{j}\left(\mathcal{B}, \ell_{0}\right)$, and (14) follows.

\section{First part of proof.}

In this section we prove the following lemma, which is a refinement of the main lemma in [7].

Lemma 2.1. Assume $\mathcal{A}$ is a $\delta$-separated subset of $G$ and for each $\ell \in \mathcal{A}$ the tube $T_{\ell}$ satisfies (3), (5).

Then for some $\sigma \in(100 \delta, 100)$ and for some subset $\mathcal{A}^{\prime} \subset \mathcal{A}$ with $\left|\mathcal{A}^{\prime}\right| \geq \delta^{C_{1} \varepsilon}|\mathcal{A}|$

$$
|E| \geq \delta^{C_{1} \varepsilon} p_{\sigma}\left(\mathcal{A}^{\prime}\right)^{-1 / 2} \lambda^{2} \sqrt{\mathcal{E}_{\delta}\left(\mathcal{A}^{\prime}\right) \mathcal{E}_{\sigma}\left(\mathcal{A}^{\prime}\right) \frac{\delta}{\sigma}} .
$$

Proof. We may assume that $\mathcal{A}$ is good in the sense of Lemma 1.4, else we pass to a suitable subset which is. (Actually, for the current argument only property 1) in the definition is needed) Let $\chi_{E}$ be the characteristic function of $E$ and define

$$
\mu_{\mathcal{A}}(x)=\sum_{m \in \mathcal{A}} \chi_{T_{m}}(x)
$$

It is easy to see that $\mu_{\mathcal{A}}(x) \lesssim \delta^{-2}$ for all $x$ - this follows since a $\delta$ separated family of lines passing through a fixed point has cardinality $\lesssim \delta^{-2}$. We also define

$$
\mu_{\mathcal{A}, \ell}^{j}(x)=\sum_{m \in \mathcal{A}: \delta^{j \varepsilon} \leq \theta(\ell, m) \leq \delta^{(j-1) \varepsilon}} \chi_{T_{m}}(x) .
$$

We claim there are positive integers $j \leq 1 / \varepsilon$ and $N \lesssim \delta^{-2}$ and a subset $\mathcal{A}^{\prime \prime} \subset \mathcal{A}$ such that

$$
\left|\mathcal{A}^{\prime \prime}\right| \geq\left(\log \frac{1}{\delta}\right)^{-2}|\mathcal{A}|
$$

and if $\ell \in \mathcal{A}^{\prime \prime}$, then

$$
\left|Y_{\ell}\right| \geq \frac{\lambda\left|T_{\ell}\right|}{\left(\log \frac{1}{\delta}\right)^{2}}
$$


where

$$
Y_{\ell} \stackrel{\text { def }}{=} T_{\ell} \cap E \cap\left\{x: \mu_{\mathcal{A}}(x) \leq 2 N\right\} \cap\left\{x: \mu_{\mathcal{A}, \ell}^{j}(x) \geq \varepsilon N\right\} .
$$

This follows from the pigeonhole principle. Namely, if $C$ is a suitable constant then for each $\ell$ there is $N$, a dyadic integer so that

$$
\left|T_{\ell} \cap E \cap\left\{x: N \leq \mu_{\mathcal{A}}(x) \leq 2 N\right\}\right| \geq \frac{\lambda\left|T_{\ell}\right|}{C \log \frac{1}{\delta}}
$$

Accordingly we can pick a value of $N$ so that (24) holds with that value of $N$ for at least $(C \log (1 / \delta))^{-1}|\mathcal{A}|$ tubes from $\mathcal{A}$. Next, for each of these tubes there must be a value of $j \leq 1 / \varepsilon$ such that

$$
\begin{aligned}
\mid T_{\ell} \cap E \cap\left\{x: N \leq \mu_{\mathcal{A}}(x) \leq 2 N\right\} \cap\left\{x: \mu_{\mathcal{A}, \ell}^{j}(x)\right. & \geq \varepsilon N\} \mid \\
& \geq \frac{\lambda\left|T_{\ell}\right|}{C \log \frac{1}{\delta}} \varepsilon
\end{aligned}
$$

and therefore (25) holds with a common value of $j$ for at least $\varepsilon(C \log (1 / \delta))^{-1}|\mathcal{A}|$ lines $\ell$. This proves the claim. We will use similar "pigeonhole" arguments several times below without giving the details.

We clearly have

$$
|E| \geq(2 N)^{-1} \sum_{\ell \in \mathcal{A}^{\prime \prime}}\left|Y_{\ell}\right| \geq \delta^{\varepsilon} \frac{\lambda \mathcal{E}_{\delta}\left(\mathcal{A}^{\prime \prime}\right)}{N} .
$$

Note that this immediately implies (18) (with $\sigma \approx \delta$ ) if $N \lambda \leq \delta^{-12 \varepsilon}$, say, so in proving (18) we may assume that $N \lambda \geq \delta^{-12 \varepsilon}$.

Assuming $N \lambda \geq \delta^{-12 \varepsilon}$ we now set $\sigma=\delta^{(j-1) \varepsilon}$ and let $\tilde{T}_{\sigma}(\ell)$ be the $3 \times 3 \sigma$ tube concentric with $T_{\ell}$. For each $\ell \in \mathcal{A}^{\prime \prime}$, we define $\mathcal{A}^{\prime \prime}(\ell)=$ $\left\{m \in \mathcal{A}^{\prime \prime}: m \sim_{j} \ell\right\}$ where $\sim_{j}$ is the relation

$$
\ell \sim_{j} m
$$

if $T_{\ell} \cap T_{m} \neq \varnothing$ and $\theta(\ell, m) \leq \delta^{(j-1) \varepsilon}$. We further define $E_{\ell}$ for $\ell \in \mathcal{A}^{\prime \prime}$ by

$$
E_{\ell}=\bigcup_{m \in \mathcal{A}^{\prime \prime}(\ell)} Y_{m}
$$

Note $E_{\ell}$ is contained in $\tilde{T}_{\sigma}(\ell)$. 
Lemma 2.2. If $N \geq \delta^{-12 \varepsilon}$ then there is a subset $\mathcal{A}^{\prime} \subset \mathcal{A}^{\prime \prime}$ with $\left|\mathcal{A}^{\prime}\right| \geq\left|\mathcal{A}^{\prime \prime}\right| / 2$, such that if $\ell \in \mathcal{A}^{\prime}$, then (with $p=p_{\sigma}(\mathcal{A})$ )

$$
\left|E_{\ell}\right| \geq \delta^{5 \varepsilon} p^{-1} N \sigma \delta \lambda^{3}
$$

Proof. Fix $\ell \in \mathcal{A}^{\prime \prime}$. If a tube $T_{m}$ intersects $T_{\ell}$ at angle greater or equal than $\delta^{\varepsilon} \sigma$ then the intersection has measure $\lesssim \delta^{-\varepsilon} \delta^{3} / \sigma$. It follows using (22) that there are at least

$$
C^{-1} \delta^{\varepsilon} N \frac{\sigma}{\delta}\left(\log \frac{1}{\delta}\right)^{-2} \lambda
$$

lines $m$ in $\mathcal{A}$ such that $T_{m}$ intersects $T_{\ell}$ at angle between $\delta^{\varepsilon} \sigma$ and $\sigma$.

Detailed justification for the latter assertion is as follows. Let $\mathcal{B}=$ $\left\{m \in \mathcal{A}: T_{m}\right.$ intersects $T_{\ell}$ at angle between $\delta^{\varepsilon} \sigma$ and $\left.\sigma\right\}$. Then

$$
\begin{aligned}
|\mathcal{B}| \delta^{-\varepsilon} \frac{\delta^{3}}{\sigma} & \gtrsim \sum_{m \in \mathcal{B}}\left|T_{m} \cap T_{\ell}\right| \\
& =\int_{T_{\ell}} \sum_{m \in \mathcal{B}} \chi_{T_{m}} \\
& =\int_{T_{\ell}} \mu_{\mathcal{A}, \ell}^{j} \\
& \geq \varepsilon N\left|Y_{\ell}\right| \\
& \gtrsim \frac{N \lambda \delta^{2}}{\left(\log \frac{1}{\delta}\right)^{2}}
\end{aligned}
$$

as claimed. We will use this argument again in Section 3 without giving the details.

By the "goodness" property, we can choose $\mathcal{A}^{\prime} \subset \mathcal{A}^{\prime \prime}$ with $\left|\mathcal{A}^{\prime}\right| \geq$ $\left|\mathcal{A}^{\prime \prime}\right| / 2$ so that if $\ell \in \mathcal{A}^{\prime}$ then there are at least

$$
C^{-1} \delta^{2 \varepsilon} N \frac{\sigma}{\delta}\left(\log \frac{1}{\delta}\right)^{-2} \lambda
$$

lines in $\mathcal{A}^{\prime \prime}$ such that $T_{m}$ intersects $T_{\ell}$ at angle less than $\sigma$, i.e.

$$
\left|\mathcal{A}^{\prime \prime}(\ell)\right| \geq C^{-1} N \delta^{2 \varepsilon} \frac{\sigma}{\delta}\left(\log \frac{1}{\delta}\right)^{-2} \lambda
$$


Fix $\ell \in \mathcal{A}^{\prime}$. We can pick $\tau \in[\delta, \sigma]$ such that at least

$$
\delta^{3 \varepsilon} N \frac{\sigma}{\delta} \lambda
$$

of these tubes $T_{m}$ intersect $T_{\ell}$ at angle between $\tau / 2$ and $\tau$. We denote this set of lines $m$ by $\mathcal{C}$. Thus

$$
|\mathcal{C}| \geq \delta^{3 \varepsilon} N \frac{\sigma}{\delta} \lambda
$$

We now repeat the argument from [7]. First we dispense with a minor technicality. Namely, we have

$$
\tau \geq \delta^{-2 \varepsilon} \delta
$$

To see this note that

$$
\delta^{3 \varepsilon} N \frac{\sigma}{\delta} \lambda \leq C \frac{\tau^{4}}{\delta^{4}},
$$

since $C \tau^{4} / \delta^{4}$ is a bound for the number of $\delta$-separated tubes which can intersect $T_{\ell}$ at angle less or equal than $\tau$. Hence, since $N \lambda \geq \delta^{-12 \varepsilon}$,

$$
\tau \geq\left(C^{-1} \delta^{3 \varepsilon} N \lambda\right)^{1 / 4}\left(\delta^{3} \sigma\right)^{1 / 4} \geq \delta^{-2 \varepsilon} \delta
$$

proving (28). Now, as in [7] we choose a family of 2 -planes $\Pi_{k}$ through $\ell$ corresponding to a maximal $(\delta / \tau)$-separated set of directions perpendicular to $\ell$ and consider their $100 \delta$-neighborhoods $\Pi_{k}^{100 \delta}$. Then every tube $T_{m}, m \in \mathcal{C}$ is contained in some $\Pi_{k}^{100 \delta}$ and a point at distance $\rho$ from $\ell$ belongs to at $\operatorname{most} C \max \{\tau / \rho, 1\} \Pi_{k}^{100 \delta}$ 's. This is clear geometrically, see also Lemma 3.1 below. For each $k$, let $\mathcal{C}_{k}$ be the tubes in $\mathcal{C}$ which are contained in $\Pi_{k}^{100 \delta}$. Let $Z_{m}$ be the points in $Y_{m}$ which are at distance at least $\delta^{\varepsilon} \tau$ from the axis $\ell$. Using (28) and standard geometrical facts, the complement of $Z_{m}$ in $Y_{m}$ is contained in a disc of radius $\approx \delta^{\varepsilon}$, so (22) and the "two ends property" (5) imply that

$$
\left|Z_{m}\right| \geq\left(\log \frac{1}{\delta}\right)^{-3} \lambda\left|T_{m}\right|
$$

Lemma 1.2 implies (using (10)) that

$$
\left|\bigcup_{m \in \mathcal{C}_{k}} Z_{m}\right| \geq \delta^{\varepsilon} p^{-1}\left|\mathcal{C}_{k}\right| \delta^{2} \lambda^{2}
$$


Therefore, since no point of any $Z_{m}$ can belong to more than $C \delta^{-\varepsilon} \Pi_{k}^{100 \delta}$ 's, we have

$$
\begin{aligned}
\left|E_{\ell}\right| & \geq\left|\bigcup_{m} Z_{m}\right| \\
& \geq \delta^{\varepsilon} \sum_{k}\left|\bigcup_{m \in \mathcal{C}_{k}} Z_{m}\right| \\
& \geq \sum_{k} \delta^{2 \varepsilon} p^{-1}\left|\mathcal{C}_{k}\right| \delta^{2} \lambda^{2} \\
& \geq \delta^{2 \varepsilon} p^{-1}|\mathcal{C}| \delta^{2} \lambda^{2} \\
& \geq \delta^{5 \varepsilon} p^{-1} N \sigma \delta \lambda^{3},
\end{aligned}
$$

by (27).

We now note the following (this is the punchline!). Let $C_{0}$ be the constant in (2).

Claim. If $x \in \mathbb{R}^{3}$, then there are at most $(2 / \varepsilon) 4 C_{0} \sigma$-separated lines $\ell \in \mathcal{A}^{\prime}$ such that $x \in E_{\ell}$.

Namely, suppose we have $M$ such lines $\ell$. For each of them there is a line $m=m_{\ell}$ at distance less or equal than $\sigma$ from $\ell$ such that $x \in Y_{m}$. Thus

1) $\mu_{\mathcal{A}}(x) \leq 2 N$.

2) $\mu_{\mathcal{A}, j}^{m}(x) \geq \varepsilon N$ for each $m$.

Note the $m$ 's are $2 C_{0} \sigma$-separated by (2), since $T_{\ell}$ intersects $T_{m_{\ell}}$ at angle less or equal than $\sigma$. It follows by (2) that no tube can intersect two different $T_{m}$ 's at angle less or equal than $\sigma$. Accordingly property 2) implies that $\mu_{\mathcal{A}}(x) \geq M \varepsilon N$, hence $M \leq 2 / \varepsilon$ by property 1$)$ This proves the claim.

Now take a maximal $4 C_{0} \sigma$-separated subset $\mathcal{B} \subset \mathcal{A}^{\prime}$. By the claim and then Lemma 2.2, we have

$$
|E| \geq \frac{2}{\varepsilon} \sum_{\ell \in \mathcal{B}}\left|E_{\ell}\right| \geq \delta^{6 \varepsilon} p^{-1}|\mathcal{B}| N \sigma \delta \lambda^{3}
$$


or in other words

$$
|E| \geq \delta^{6 \varepsilon} p^{-1} N \lambda^{3} \mathcal{E}_{\sigma}\left(\mathcal{A}^{\prime}\right) \frac{\delta}{\sigma}
$$

since of course $\mathcal{E}_{\sigma}(\mathcal{A})$ and $\mathcal{E}_{4 C_{0} \sigma}(\mathcal{A})$ are comparable. If we take the geometric mean of (29) and (26) we get (18).

A slab of thickness $\phi$ is a $\phi$-neighborhood of a 2-plane. What we actually use below is the following corollary of Lemma 2.1 .

Lemma 2.3. Assume $\mathcal{A}$ is a $\delta$-separated subset of $G$ and for each $\ell \in \mathcal{A}$ the tube $T_{\ell}$ satisfies (3) and (5).

Assume in addition that all tubes $T_{\ell}, \ell \in \mathcal{A}$ are contained in a slab of thickness $\phi$ and in a $\rho$-neighborhood of a line. Let $p=p_{\rho}(\mathcal{A})$ and define $m=m(\mathcal{A})$ via

$$
\begin{aligned}
& m(\mathcal{A})=\max _{e \in S^{2}} m(\mathcal{A}, e), \\
& \text { where } m(\mathcal{A}, e) \stackrel{\text { def }}{=}\left|\left\{\ell \in \mathcal{A}: \theta\left(e, \ell^{*}\right)<\delta\right\}\right| .
\end{aligned}
$$

Then

$$
|E| \geq \delta^{C_{2} \varepsilon}(m p)^{-1 / 2} \lambda^{2} \mathcal{E}_{\delta}(\mathcal{A}) \sqrt{\frac{\delta}{\phi}} .
$$

Proof. Fix a number $\sigma \geq \delta$. Note that all the lines in $\mathcal{A}$ make an angle less or equal than $\phi$ with a fixed 2-plane. We will use this fact to get a lower bound on $\mathcal{E}_{\sigma}(\mathcal{A})$. Namely, let $\mathcal{A}^{*}$ be the set of angles $\ell^{*}, \ell \in \mathcal{A}$. Clearly

$$
\mathcal{E}_{\delta}\left(\mathcal{A}^{*}\right) \gtrsim \frac{\mathcal{E}_{\delta}(\mathcal{A})}{m}
$$

On the other hand, $\mathcal{A}^{*}$ is contained in a $\phi$-neighborhood of a great circle on the 2-sphere, which implies that

$$
\mathcal{E}_{\sigma}\left(\mathcal{A}^{*}\right) \gtrsim \frac{\sigma}{\phi} \mathcal{E}_{\phi}\left(\mathcal{A}^{*}\right)
$$

when $\sigma \geq \phi$. Also $\mathcal{E}_{\sigma}\left(\mathcal{A}^{*}\right) \gtrsim \mathcal{E}_{\tau}\left(\mathcal{A}^{*}\right)$ if $\sigma \geq \tau$ (this is true for any set on the 2 -sphere), so we may conclude that

$$
\frac{\mathcal{E}_{\sigma}\left(\mathcal{A}^{*}\right)}{\sigma} \gtrsim \frac{\mathcal{E}_{\delta}\left(\mathcal{A}^{*}\right)}{\phi},
$$


for all $\sigma$, and therefore

$$
\frac{\mathcal{E}_{\sigma}\left(\mathcal{A}^{*}\right)}{\sigma} \gtrsim \frac{\mathcal{E}_{\delta}(\mathcal{A})}{m \phi}
$$

The result now follows from Lemma 2.1.

Corollary. Under the assumptions of Lemma 2.3, suppose that for each $\ell \in \mathcal{A}$ a subset $Y_{\ell} \subset T_{\ell} \cap E$ is given, with

$$
\left|Y_{\ell}\right| \geq\left(\log \frac{1}{\delta}\right)^{-4} \lambda\left|T_{\ell}\right|
$$

Let $\tilde{E}=\cup_{\ell \in \mathcal{A}} Y_{\ell}$. Then estimate (31) holds also for $\tilde{E}$, i.e.

$$
|\tilde{E}| \geq \delta^{C_{2} \varepsilon}(m p)^{-1 / 2} \lambda^{2} \mathcal{E}_{\delta}(\mathcal{A}) \sqrt{\frac{\delta}{\phi}} .
$$

Proof. The idea is to apply Lemma 2.3 with $E$ replaced by $\tilde{E}$ and $\lambda$ replaced by

$$
\lambda\left(\log \frac{1}{\delta}\right)^{-4} .
$$

In order to do this we must make the following remarks:

- (5) does not quite hold anymore. However, it holds if we replace the exponent 10 on the right hand side by 6 . The reader can easily check that this does not make any difference.

- The definition of the number $p_{\sigma}(\mathcal{A})$ depended in principle on $\lambda$ and $E$ as well as $\mathcal{A}$. However, in fact it depends only on $\mathcal{A}$ by the corollary to Lemma 1.1.

Accordingly we can apply Lemma 2.3 as indicated, obtaining

$$
|\tilde{E}| \geq \delta^{C_{2} \varepsilon}(m p)^{-1 / 2} \lambda^{2}\left(\log \frac{1}{\delta}\right)^{-8} \mathcal{E}_{\delta}(\mathcal{A}) \sqrt{\frac{\delta}{\phi}} .
$$

The factor $(\log (1 / \delta))^{-8}$ may of course be incorporated into the $\delta^{C_{2} \varepsilon}$ factor, so we are done.

REMARK. The considerations in Section 2 generalize immediately to higher dimensions. In particular, Lemma 2.1 is true in $\mathbb{R}^{n}$ with the same 
proof provided we define $\mathcal{E}_{\sigma}(\mathcal{A})=\sigma^{n-1}$ times the maximum possible cardinality for a $\sigma$-separated subset of $\mathcal{A}$, define $p$ using $100 \times w \times$ $100 \delta \times \cdots \times 100 \delta$ rectangles and replace the factor $\delta / \sigma$ by $(\delta / \sigma)^{n-2}$.

\section{Main argument.}

The argument in this section will be based on considering families of tubes which intersect a plate, rather than a tube as in the previous section. Lemmas 3.1 and 3.2 below record some geometrical facts relevant in this situation.

Lemma 3.1. Suppose that $\phi \in(\delta, \pi / 2), \sigma \in(\delta, \pi / 2), w \leq \sigma$ and $R$ is a $100 \times w \times 100 \delta$ rectangle. Let $\Pi$ be the 2 -plane of $R$, let $\left\{\theta_{k}\right\}$ be a maximal $(\phi w+\delta) / \sigma$-separated subset of $(\phi / 2, \phi)$, and for each $k$ let $\Pi_{k}^{ \pm}$be the two 2-planes through the axis of $R$ which make an angle $\theta_{k}$ with $\Pi$, and $\Pi_{k}^{ \pm, C(\phi w+\delta)}$ their $C(\phi w+\delta)$-neighborhoods. Then:

i) Let $T_{\ell}$ be a tube which intersects $R$ and such that $\ell$ makes an angle less or equal than $\sigma$ with the axis of $R$ and the 2-plane spanned by $\ell$ and the axis direction of $R$ makes an angle between $(\phi-\delta) / 2$ and $\phi$ with the 2-plane $\Pi$. Then $T_{\ell}$ is contained in some slab $\Pi_{k}^{ \pm, C(\phi w+\delta)}$.

ii) A point at distance greater or equal than $\rho$ from $\Pi$ is contained in $\lesssim \max \{\phi \sigma / \rho, 1\}$ slabs $\Pi_{k}^{ \pm, C(\phi w+\delta)}$.

Proof. i) First let $\Pi_{1}$ and $\Pi_{2}$ be 2-planes passing through the axis of $R$ and making angle less or equal than $\beta$ with each other. Let $\tau_{\sigma}$ be the $\sigma$-neighborhood of the axis of $R$. Then every point of $\tau_{\sigma} \cap \Pi_{2}$ will be within $\beta \sigma$ of $\Pi_{1}$. Accordingly (take $\beta=(\phi w+\delta) / \sigma$ ) it suffices to show that $T_{\ell}$ is contained in a $C(\phi w+\delta)$-neighborhood of some plane passing through the axis of $R$ and making an angle between $\phi / 2$ and $\phi$ with $\Pi$. On the other hand, let $\Pi^{\prime}$ be the plane spanned by $\ell$ and the axis direction of $R$. Let $\Pi^{\prime \prime}$ be the plane parallel to $\Pi^{\prime}$ which passes through the axis of $R$. The distance between $\Pi^{\prime}$ and $\Pi^{\prime \prime}$ is then $\lesssim \phi w+\delta$ and therefore $T_{\ell}$ is contained in the $C(\phi w+\delta)$-neighborhood of $\Pi^{\prime \prime}$.

ii) Choose coordinates so that $\Pi$ is the $x y$ plane and the axis of

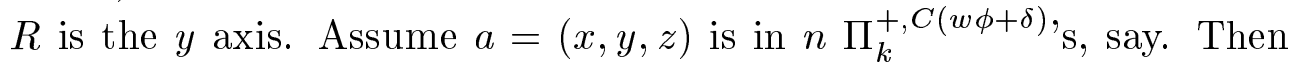
(assuming $\phi \leq \pi / 4$; otherwise some minor changes in the argument are 
required) we have

$$
|z|=\left(\sin \theta_{k}\right)|x|+\mathcal{O}(\phi w+\delta),
$$

for each $k$ and therefore, arranging the $\theta_{k}$ 's in increasing order,

$$
(n-1) \frac{\phi w+\delta}{\sigma}|x| \leq\left(\theta_{n}-\theta_{1}\right)|x| \lesssim(\phi w+\delta),
$$

so $|x| \lesssim \sigma /(n-1)$. Then

$$
|z| \lesssim \frac{\phi \sigma}{n-1}+\phi w+\delta
$$

which implies

$$
|z| \lesssim \frac{\phi \sigma}{n-1}
$$

since obviously

$$
n-1 \lesssim \frac{\phi \sigma}{\phi w+\delta}
$$

This is equivalent to the statement.

Lemma 3.2. Suppose that $\sigma \in(\delta, \pi / 2), \phi \in(\delta, \pi / 2)$ and $R$ is a $100 \times w \times 100 \delta$ rectangle. Let $\ell$ be a line and assume that $\ell$ makes an angle greater or equal than $\sigma-\delta$ with the axis direction of $R$ and that the 2-plane spanned by $\ell$ and the axis direction of $R$ makes an angle greater or equal than $\phi-\delta$ with the 2 -plane of $R$. Then

$$
\left|T_{\ell} \cap R\right| \lesssim \min \left\{\delta^{2} \frac{w}{\sigma}, \frac{\delta^{3}}{\phi \sigma+\delta}\right\}
$$

Proof. Choose coordinates so the axis direction of $R$ is the $y$ direction, the 2-plane of $R$ is parallel to the $x y$ plane and the origin belongs to $T_{\ell} \cap R$. If $p=(x, y, z)$ is a point of $T_{\ell}$ then the assumptions mean that

$$
\begin{aligned}
& |x|+|y|+|z| \lesssim \sigma^{-1}(|x|+|z|)+\delta, \\
& |x|+|z| \lesssim \phi^{-1}|z|+\delta
\end{aligned}
$$

and therefore

$$
|x|+|y|+|z| \lesssim(\phi \sigma)^{-1}|z|+\sigma^{-1} \delta
$$


If $p \in T_{\ell} \cap R$ then (32) and (33) imply

$$
|x|+|y|+|z| \lesssim \min \left\{\sigma^{-1}(w+\delta),(\phi \sigma)^{-1} \delta\right\},
$$

or in other words $T_{\ell} \cap R$ is a subset of $T_{\ell}$ with diameter

$$
\lesssim \min \left\{\frac{w}{\sigma}, \frac{\delta}{\phi \sigma}\right\}
$$

The lemma follows.

The next lemma estimates the measure of the union of a "large" family of tubes intersecting a rectangle.

Lemma 3.3. Suppose $\sigma \geq 100 \delta, \phi \in(\delta, \pi / 2), w \leq \sigma, R$ is a $100 \times$ $w \times 100 \delta$ rectangle and $\mathcal{C}$ is a family of lines. Assume that if $\ell \in \mathcal{C}$ then $T_{\ell}$ intersects $R, \ell$ makes an angle less or equal than $\sigma$ with the axis direction of $R$, and the 2-plane spanned by $\ell$ and the axis direction of $R$ makes an angle in $((\phi-\delta) / 2, \phi)$ with the 2-plane of $R$. Assume furthermore that if $\ell \in \mathcal{C}$ then $T_{\ell}$ satisfies (3), (5). Let $p=p_{\sigma}(\mathcal{C})$ and define $m=m(\mathcal{C})$ via (30). Assume that for each $\ell \in \mathcal{C}$ a subset $Y_{\ell} \subset T_{\ell} \cap E$ is given, with

$$
\left|Y_{\ell}\right| \geq\left(\log \frac{1}{\delta}\right)^{-3} \lambda\left|T_{\ell}\right|
$$

Let $\tilde{E}=\cup_{\ell} Y_{\ell}$. Then $\tilde{E}$ is contained in a slab of width $C(\phi \sigma+\delta)$ and

$$
|\tilde{E}| \geq \delta^{C_{3} \varepsilon}(m p)^{-1 / 2} \lambda^{2} \mathcal{E}_{\delta}(\mathcal{C}) \sqrt{\frac{\delta}{w \phi+\delta}}
$$

Proof. It follows by Lemma 1.0 that $\tilde{E}$ is contained in a slab of width $C(\phi \sigma+\delta)$ - namely, the $C(\phi \sigma+\delta)$-neighborhood of the 2-plane of $R$. We now prove (34). We first dispense with a couple of minor technicalities. First of all, we can assume that all the lines in $\mathcal{C}$ actually make an angle between $\delta^{-\varepsilon} \sigma$ and $\sigma$ with the axis direction of $R$, since we can always achieve this by replacing $\sigma$ by $\delta^{j \varepsilon} \sigma$ for a suitable $j \geq 0$ and replacing $\mathcal{C}$ by a subset $\mathcal{C}^{\prime}$ with $\mathcal{E}_{\delta}\left(\mathcal{C}^{\prime}\right) \gtrsim \mathcal{E}_{\delta}(\mathcal{C})$. Second, we can assume $\phi \sigma \geq \delta^{-3 \varepsilon} \delta$. To see this, suppose that $\phi \sigma \leq \delta^{-3 \varepsilon} \delta$. Then all the tubes in $\mathcal{C}$ are contained in a $C \delta^{1-3 \varepsilon}$-neighborhood of a 2-plane. Accordingly (34) follows immediately from the corollary to Lemma 2.3. 
Now we consider the main case where $\phi \sigma \geq \delta^{-3 \varepsilon} \delta$. Let $Z_{\ell}$ be the points in $Y_{\ell}$ which are at distance greater or equal than $\delta^{2 \varepsilon} \phi \sigma$ from the 2-plane of $R$ and $\tilde{\tilde{E}}=\cup_{\ell} Z_{\ell}$. Since $\phi \sigma \geq \delta^{-3 \varepsilon} \delta$, it follows from (33) that the set of points of $T_{\ell}$ which are within $\delta^{2 \varepsilon} \phi \sigma$ of the 2-plane of $R$ is contained in a $C \delta^{\varepsilon}$-disc. Thus the complement of $Z_{\ell}$ in $Y_{\ell}$ is contained in a $C \delta^{\varepsilon}$-disc. So property (5) implies

$$
\left|Z_{\ell}\right| \geq \lambda\left(\log \frac{1}{\delta}\right)^{-4}\left|T_{\ell}\right|
$$

Now consider a subdivision into 2-plane neighborhoods $\Pi_{k}^{C(w \phi+\delta)}$ as in Lemma 3.1, relative to the rectangle $R$, and with the given value of $\sigma$. Let $\mathcal{C}_{k}$ be the tubes which are contained in a given $\Pi_{k}^{C(\phi w+\delta)}$. By the corollary to Lemma 2.3,

$$
\left|\tilde{\tilde{E}} \cap \Pi_{k}^{C(w \phi+\delta)}\right| \geq \delta^{C_{2} \varepsilon}(m p)^{-1 / 2} \lambda^{2} \mathcal{E}_{\delta}\left(\mathcal{C}_{k}\right) \sqrt{\frac{\delta}{w \phi+\delta}} .
$$

Notice that no point of $\tilde{\tilde{E}}$ is in more than $C \delta^{-\varepsilon}$ sets of the form $\tilde{\tilde{E}} \cap$ $\Pi_{k}^{C(w \phi+\delta)}$, by Lemma 3.1.ii). So if we sum over $k$ we get

$$
\begin{aligned}
|\tilde{E}| & \geq|\tilde{\tilde{E}}| \\
& \geq \delta^{\varepsilon} \sum_{k}\left|\tilde{\tilde{E}} \cap \Pi_{k}^{C(w \phi+\delta)}\right| \\
& \geq \delta^{C \varepsilon} \sum_{k}(m p)^{-1 / 2} \lambda^{2} \mathcal{E}_{\delta}\left(\mathcal{C}_{k}\right) \sqrt{\frac{\delta}{w \phi+\delta}} \\
& \geq \delta^{C \varepsilon}(m p)^{-1 / 2} \lambda^{2} \mathcal{E}_{\delta}(\mathcal{C}) \sqrt{\frac{\delta}{w \phi+\delta}} .
\end{aligned}
$$

In order to apply Lemma 3.3 we need to find sufficiently large families of tubes which intersect a suitable rectangle. This is done in the next lemma, which is analogous to Lemma 2.2 in Section 2. The quantities $\mu_{\mathcal{A}}, \mu_{\mathcal{A}, \ell}^{j}$ were defined in (19), (20).

Lemma 3.4. Assume that $\mathcal{A} \subset G$ is $\delta$-separated and that the tubes $T_{\ell}$ satisfy (3) and (5), and furthermore that $\mathcal{A}$ is good in the sense of Lemma 1.4. Fix $j$ and suppose that $\mathcal{B}$ is a subset of $\mathcal{A}$ with

$$
|\mathcal{B}| \geq\left(\log \frac{1}{\delta}\right)^{-10}|\mathcal{A}|
$$


and that for each $\ell \in \mathcal{B}$, a subset

$$
Y_{\ell} \subset T_{\ell} \cap E \cap\left\{x: \mu_{\mathcal{A}}(x) \leq 2 N\right\} \cap\left\{x: \mu_{\mathcal{A}, \ell}^{j}(x) \geq \varepsilon N\right\}
$$

is given, with

$$
\left|Y_{\ell}\right| \geq\left(\log \frac{1}{\delta}\right)^{-3} \lambda\left|T_{\ell}\right|
$$

Let $\sigma=\delta^{(j-1) \varepsilon}$ and let $m=m(\mathcal{A})$. Then for some line $\ell \in \mathcal{B}$ there are a number $\phi \in(\delta, \pi / 2)$, a 2-plane $\Pi$ and a set of lines $\mathcal{D} \subset \mathcal{B} \cap$ $D\left(\ell, C_{4} \delta^{-\varepsilon} \sigma\right)$ such that

$$
\bigcup_{m \in \mathcal{D}} Y_{m} \subset \Pi^{C\left(\delta^{-2 \varepsilon} \phi \sigma+\delta\right)}
$$

and

$$
\left|\bigcup_{m \in \mathcal{D}} Y_{m}\right| \geq \delta^{C_{5} \varepsilon} N m^{-1 / 2} \lambda^{7 / 2} \delta \sqrt{\sigma} \sqrt{\phi \sigma+\delta} .
$$

Proof. Let $\mathcal{C}$ be associated to $\mathcal{B}$ as in the definition of "good" preceding Lemma 1.4. We will show that the conclusion holds for any $\ell \in \mathcal{C}$. So fix a line $\ell_{0} \in \mathcal{C}$.

By (14) and part ii) of the corollary to Lemma 1.1, $T_{\ell_{0}}$ must be contained in a plate $P$ relative to $\mathcal{B}$ of width $w \leq \max \left\{100 \delta, \delta^{2 \varepsilon} \sigma\right\}$ and $\mathcal{B}$-tube density $d_{\mathcal{B}}(P) \geq \delta^{7 \varepsilon} p$, where $p=p_{\sigma}(\mathcal{A})$.

Claim. For some $\phi \geq \delta$, there is a set $\mathcal{D}_{0} \subset \mathcal{A}$ with

$$
\left|\mathcal{D}_{0}\right| \gtrsim \delta^{12 \varepsilon} N\left(p \frac{w}{\delta}\right)^{1 / 2} \lambda^{3 / 2} \max \left\{\delta^{-1}(\phi \sigma+\delta), \frac{\sigma}{w}\right\}
$$

such that if $\ell \in \mathcal{D}_{0}$ then $T_{\ell}$ intersects $P, \ell$ makes an angle less or equal than $\sigma$ with the axis of $P$, and the 2-plane spanned by $\ell$ and the axis direction of $P$ makes an angle less or equal than $\phi$ with the 2-plane of $P$.

To prove the claim, let $\Sigma$ be the set of lines $\ell \in \mathcal{B}$ such that $T_{\ell} \subset P$ and $Z=\cup\left\{Y_{\ell}: \ell \in \Sigma\right\}$. Then, since $|\Sigma| \geq \delta^{7 \varepsilon} p w / \delta$, we have

$$
|Z| \gtrsim \delta^{8 \varepsilon} \lambda^{2} \delta w
$$


by Lemma 1.2. We will now show that also

$$
|Z| \gtrsim \delta^{8 \varepsilon} \lambda \delta^{2} p
$$

Namely, let $\Sigma^{*}$ be the set of directions of lines in $\Sigma$. It is clear that the maximum possible cardinality for a $\delta$-separated subset of $\Sigma^{*}$ is $\lesssim w / \delta$. Accordingly, by definition of $d_{\mathcal{B}}(P)$ there must be a direction $e$ such that $\theta\left(\ell^{*}, e\right)<\delta$ for $\gtrsim \delta^{7 \varepsilon} p$ lines $\ell \in \Sigma$. Denote this set of $\gtrsim \delta^{7 \varepsilon} p$ lines by $\Sigma^{\prime}$. It is clear that no point can belong to more than a bounded number of the (essentially parallel) tubes $T_{\ell}, \ell \in \Sigma^{\prime}$. Accordingly

$$
|Z| \gtrsim \sum_{\ell \in \Sigma^{\prime}}\left|Y_{\ell}\right| \gtrsim \delta^{6 \varepsilon} p\left(\log \frac{1}{\delta}\right)^{-3} \lambda \delta^{2}
$$

and (37) follows.

Taking the geometric mean of (36) and (37) we conclude that

$$
|Z| \gtrsim \delta^{8 \varepsilon} \sqrt{\frac{p w}{\delta}} \lambda^{3 / 2} \delta^{2}
$$

Next, each point $x \in Z$ belongs to $Y_{m}$ for some line $m$ such that $T_{m} \subset P$. By definition of $Y_{m}$ there are $\gtrsim N$ lines $\ell \in \mathcal{A}$ such that $T_{\ell}$ contains $x$ and $\ell$ makes an angle between $\delta^{\varepsilon} \sigma$ and $\sigma$ with the line $m$. We denote this set of lines $\ell$ by $\mathcal{A}(x)$. Since the width of $P$ is less or equal than $\max \left\{\delta^{2 \varepsilon} \sigma, 100 \delta\right\}$ it follows that all lines $\ell \in \cup_{x \in Z} \mathcal{A}(x)$ make an angle between $\delta^{\varepsilon} \sigma / 2-C \delta$ and $2 \sigma+C \delta$ with the axis of $P$. For each $\ell \in$ $\cup_{x \in Z} \mathcal{A}(x)$, the 2-plane spanned by $\ell$ and the axis direction of $P$ makes a certain angle $\phi_{\ell}$ depending on $\ell$ with the 2-plane of $P$. We can now use the pigeonhole principle to obtain a common value of $\phi_{\ell}$. Namely, by the pigeonhole principle there are a number $\phi \in(\delta, \pi / 2)$ and a subset $F \subset Z$ with $|F| \geq \delta^{\varepsilon}|Z|$, so that if $x \in F$ then there is a subset $\tilde{\mathcal{A}}(x) \subset \mathcal{A}(x)$ with cardinality at least $N \delta^{\varepsilon}$, which consists of lines $\ell$ such that $\phi_{\ell} \in((\phi-\delta) / 2, \phi)$.

To summarize: there is a subset $F \subset P$ with measure greater or equal than

$$
\delta^{9 \varepsilon} \sqrt{\frac{p w}{\delta}} \lambda^{3 / 2} \delta^{2}
$$

so that if $x \in F$ then there is a set (which we denoted $\tilde{\mathcal{A}}(x)$ ) of $N \delta^{\varepsilon}$ lines $\ell \in \mathcal{A}$ such that $T_{\ell}$ contains $x$ and $\ell$ makes an angle in $\left(\delta^{\varepsilon} \sigma / 2-\right.$ $C \delta, 2 \sigma+C \delta$ ) with the axis of $P$ and the 2-plane spanned by $\ell$ and the 
axis direction of $P$ makes an angle in $((\phi-\delta) / 2, \phi)$ with the 2-plane of $P$. We define $\mathcal{D}_{0}=\cup_{x \in F} \tilde{\mathcal{A}}(x)$. By Lemma 3.2, we have

$$
\left|T_{\ell} \cap P\right| \lesssim \delta^{-2 \varepsilon} \min \left\{\frac{\delta^{3}}{\phi \sigma+\delta}, \delta^{2} \frac{w}{\sigma}\right\}
$$

for any $\ell \in \mathcal{D}_{0}$. From this we conclude in a standard way (see the argument at the beginning of the proof of Lemma 2.2 that the cardinality of $\mathcal{D}_{0}$ must be

$$
\gtrsim \delta^{12 \varepsilon} N\left(p \frac{w}{\delta}\right)^{1 / 2} \lambda^{3 / 2} \max \left\{\delta^{-1}(\phi \sigma+\delta), \frac{\sigma}{w}\right\},
$$

proving the claim.

It follows by (15) in the definition of good (applied with $\delta^{i \varepsilon}=\delta^{-\varepsilon} \sigma$, $r$ as large as possible subject to $\delta^{r \varepsilon} \geq \phi$, and $j$ as large as possible subject to $\left.\delta^{j \varepsilon} \geq w\right)$ that there is a plate $P^{\prime}$ containing $T_{\ell_{0}}$ with width $w^{\prime} \leq \delta^{-\varepsilon} w$ which intersects at least

$$
\delta^{13 \varepsilon} N\left(p \frac{w}{\delta}\right)^{1 / 2} \lambda^{3 / 2} \max \left\{\delta^{-1}(\phi \sigma+\delta), \frac{\sigma}{w}\right\}
$$

tubes $T_{\ell}$ with $\ell \in \mathcal{B}$ such that $\ell$ makes an angle less or equal than $\delta^{-\varepsilon} \sigma$ with the axis of $P^{\prime}$ and the 2-plane spanned by $\ell$ and the axis direction of $P^{\prime}$ makes an angle less or equal than $\delta^{-\varepsilon} \phi$ with the 2-plane of $P^{\prime}$. We can pigeonhole to obtain a number $\tau \leq \delta^{-\varepsilon} \phi$ and a choice of

$$
\delta^{14 \varepsilon} N\left(p \frac{w}{\delta}\right)^{1 / 2} \lambda^{3 / 2} \max \left\{\delta^{-1}(\phi \sigma+\delta), \frac{\sigma}{w}\right\},
$$

of these tubes $T_{\ell}$ for which the 2-plane spanned by $\ell$ and the axis direction of $P^{\prime}$ makes an angle in $((\tau-\delta) / 2, \tau)$ with the 2-plane of $P^{\prime}$, and we let $\mathcal{D}$ be the lines $\ell$ corresponding to the latter set of tubes $T_{\ell}$. It is easy to see that $\mathcal{D} \subset D\left(\ell_{0}, C_{4} \delta^{-\varepsilon} \sigma\right)$ : this follows since i) each tube in $\mathcal{D}$ intersects the plate $P^{\prime}$ at angle less or equal than $\delta^{-\varepsilon} \sigma$ to its axis and therefore (since $w^{\prime} \leq \delta^{-\varepsilon} \sigma$ ) also at angle $\lesssim \delta^{-\varepsilon} \sigma$ to the direction of $\ell_{0}$ and ii) since $w^{\prime} \leq \delta^{-\varepsilon} \sigma$, every point of $P^{\prime}$ is within $C \delta^{-\varepsilon} \sigma$ of $\ell_{0}$. It remains to observe that $\cup_{m \in \mathcal{C}} Y_{m} \subset \Pi^{C\left(\delta^{-2 \varepsilon} \phi \sigma+\delta\right)}$ where $\Pi$ is the 2 -plane of $P^{\prime}$ and to prove (35). For this we apply Lemma 3.3, with $R$ there equal to $P^{\prime}$ and $\sigma$ there replaced by $\delta^{-\varepsilon} \sigma$ and $\phi$ there equal to $\tau$. We conclude in the first place that

$$
\bigcup_{m \in \mathcal{D}} Y_{m} \subset \Pi^{C\left(\tau \delta^{-\varepsilon} \sigma+\delta\right)} \subset \Pi^{C\left(\delta^{-\varepsilon} \phi \delta^{-\varepsilon} \sigma+\delta\right)} .
$$


Note also that $p_{\delta^{-\varepsilon} \sigma}(\mathcal{D}) \leq \delta^{-C \varepsilon} p_{\sigma}(\mathcal{D}) \leq \delta^{-C \varepsilon} p$ by the corollary to Lemma 1.1 and then (10). Hence by (34),

$$
\begin{aligned}
\left|\bigcup_{m \in \mathcal{C}} Y_{m}\right| \geq & \delta^{C \varepsilon}(p m)^{-1 / 2} \lambda^{2}\left(N\left(p \frac{w}{\delta}\right)^{1 / 2} \lambda^{3 / 2} \max \left\{\delta^{-1}(\phi \sigma+\delta), \frac{\sigma}{w}\right\} \delta^{2}\right) \\
& \cdot \sqrt{\frac{\delta}{w^{\prime} \tau+\delta}} \\
\geq & \delta^{C \varepsilon}(p m)^{-1 / 2} \lambda^{2}\left(N\left(p \frac{w}{\delta}\right)^{1 / 2} \lambda^{3 / 2} \max \left\{\delta^{-1}(\phi \sigma+\delta), \frac{\sigma}{w}\right\} \delta^{2}\right) \\
& \cdot \sqrt{\frac{\delta}{w \phi+\delta}} \\
= & \delta^{C \varepsilon} N m^{-1 / 2} \lambda^{7 / 2}\left(\frac{w}{\delta}\right)^{1 / 2} \max \left\{\delta^{-1}(\phi \sigma+\delta), \frac{\sigma}{w}\right\} \delta^{2} \\
& \cdot \sqrt{\frac{\delta}{w \phi+\delta}} \geq \\
\geq & \delta^{C \varepsilon} N m^{-1 / 2} \lambda^{7 / 2} \delta \sqrt{\sigma} \sqrt{\phi \sigma+\delta} .
\end{aligned}
$$

Inequality (38) may be seen as follows: if $w \phi \geq \delta$ then

$$
\begin{aligned}
\left(\frac{w}{\delta}\right)^{1 / 2} \max \left\{\delta^{-1}(\phi \sigma+\delta), \frac{\sigma}{w}\right\} \delta^{2} & \sqrt{\frac{\delta}{w \phi+\delta}} \\
& \gtrsim\left(\frac{w}{\delta}\right)^{1 / 2} \delta^{-1}(\phi \sigma+\delta) \delta^{2} \sqrt{\frac{\delta}{w \phi}} \\
& \geq \delta \sqrt{\sigma} \sqrt{\phi \sigma+\delta} .
\end{aligned}
$$

On the other hand if $w \phi \leq \delta$ then

$$
\begin{aligned}
\left(\frac{w}{\delta}\right)^{1 / 2} \max \left\{\delta^{-1}(\phi \sigma+\delta), \frac{\sigma}{w}\right\} \delta^{2} & \sqrt{\frac{\delta}{w+\delta}} \\
& \gtrsim\left(\frac{w}{\delta}\right)^{1 / 2} \delta^{2} \sqrt{\delta^{-1}(\phi \sigma+\delta) \frac{\sigma}{w}} \\
& \geq \delta \sqrt{\sigma} \sqrt{\phi \sigma+\delta}
\end{aligned}
$$

This proves (38), hence (35). 
Now we need a simple lemma. Here we let

$$
f_{\delta}^{*}(e)=\sup _{\ell: \ell^{*}=e} \sup _{a \in \ell} \frac{1}{\left|T_{\ell}(a)\right|} \int_{T_{\ell}(a)} f
$$

be the Kakeya maximal function as defined in [1].

Lemma 3.5. Suppose that $\delta \leq \sigma \leq 100$ and that $\left\{S_{j}\right\}_{j=1}^{M}$ are slabs with respective thicknesses less or equal than $C\left(\phi_{j} \sigma+\delta\right)$. Let $f=\sum_{j} \chi_{S_{j}}$. Fix $e_{0} \in S^{2}$. Then

$$
\left|\left\{e \in D\left(e_{0}, \sigma\right): f_{\delta}^{*}(e) \geq \lambda\right\}\right| \lesssim \frac{\sigma \sum_{j}\left(\phi_{j} \sigma+\delta\right)}{\lambda} \log \frac{1}{\delta}
$$

ProOF. If $\sum_{j=1}^{M}\left(\phi_{j} \sigma+\delta\right) \geq \sigma$ there is nothing to prove. It follows that we can assume $M \leq 1 / \delta$.

First consider the case where there is just one slab $S$, with thickness $\lesssim \phi \sigma+\delta$. Then the set $\left\{e \in D\left(e_{0}, \sigma\right): f_{\delta}^{*} \geq \lambda\right\}$ is contained in the intersection of $D\left(e_{0}, \sigma\right)$ with a $(C(\phi \sigma+\delta) / \lambda)$-neighborhood of a great circle, so its measure is $\lesssim \sigma(\phi \sigma+\delta) / \lambda$. Since $M \leq 1 / \delta$, the general case now follows from the Stein - N. J. Weiss result on summing weak type 1 estimates.

In the next corollary we use the notation $\ell^{*}=$ direction of the line $\ell$, and if $\mathcal{C}$ is a set of lines then $\mathcal{C}^{*}=\left\{\ell^{*}\right\}_{\ell \in \mathcal{C}}$.

Corollary. Let $\left\{E_{k}\right\}_{k=1}^{M}$ be a family of subsets of the unit ball in $\mathbb{R}^{3}$, such that $E_{k}$ is contained in a $C\left(\phi_{k} \sigma+\delta\right)$-slab. Let $\mathcal{C}$ be a family of lines and assume that for each $\ell \in \mathcal{C}$ a subset $\mathcal{K}(\ell) \subset\{1, \ldots, M\}$ is given, and that the following holds

$$
\text { If } \operatorname{dist}\left(\ell_{1}^{*}, \ell_{2}{ }^{*}\right) \geq C \sigma, \quad \text { then } \mathcal{K}\left(\ell_{1}\right) \cap \mathcal{K}\left(\ell_{2}\right)=\varnothing \text {. }
$$

Let $E(\ell)=\cup_{k \in \mathcal{K}(\ell)} E_{k}$, and assume that for every $\ell \in \mathcal{C}$, we have $\mid T_{\ell} \cap$ $E(\ell)\left|\geq \delta^{\varepsilon} \lambda\right| T_{\ell} \mid$. Then

$$
\sigma \sum_{k}\left(\phi_{k} \sigma+\delta\right) \geq \delta^{2 \varepsilon} \lambda \mathcal{E}_{\delta}\left(\mathcal{C}^{*}\right)
$$


Proof. Because of property (39) it suffices to show (40) assuming that $\mathcal{C}^{*}$ is contained in a single $\sigma$-disc, and in that case it is immediate from Lemma 3.5 since $\left(\sum_{k} \chi_{E_{k}}\right)^{*} \gtrsim \delta^{\varepsilon} \lambda$ on a $\delta$-neighborhood of $\mathcal{C}^{*}$.

Proof of Lemma 0 . We start by fixing a maximal $\delta$-separated subset $\Sigma^{*}$ of $\Omega$, and for each $e \in \Sigma^{*}$ we choose (exactly) $m \delta$-separated lines $\ell$ with $\ell^{*}=e$ and so that the tubes $T_{\ell}$ satisfy (3) and (5). We then choose a "good" subset by Lemma 1.4. We denote this last set of lines by $\mathcal{A}$. Note that

$$
\mathcal{E}_{\delta}(\mathcal{A}) \geq \delta^{\varepsilon} m|\Omega|
$$

and also

$$
\mathcal{E}_{\delta}\left(\mathcal{C}^{*}\right) \geq \delta^{2 \varepsilon}|\Omega|
$$

if $\mathcal{C}$ is any subset of $\mathcal{A}$ with $|\mathcal{C}| \geq \delta^{\varepsilon}|\mathcal{A}|$. Furthermore, the quantity $m(\mathcal{A})$ defined by $(30)$ is $\lesssim m$.

We choose $N$ and $\sigma=\delta^{(j-1) \varepsilon}$ as in the proof of Lemma 2.1 so that the set

$$
Y_{\ell}^{0} \stackrel{\text { def }}{=} T_{\ell} \cap E \cap\left\{x: \mu_{\mathcal{A}}(x) \leq 2 N\right\} \cap\left\{x: \mu_{\mathcal{A}, \ell}^{j}(x) \geq \varepsilon N\right\}
$$

will have measure greater or equal than

$$
\left(\log \frac{1}{\delta}\right)^{-2} \lambda\left|T_{\ell}\right|
$$

for a set of $\ell \in \mathcal{A}$ with cardinality greater or equal

$$
\left(\log \frac{1}{\delta}\right)^{-2}|\mathcal{A}|
$$

and we let $\mathcal{B}$ be this set of $\ell^{\prime}$ s. We also let $\left\{\ell_{j}\right\}$ be a maximal $\delta^{-\varepsilon} \sigma$ separated subset of $\mathcal{B}$ and let $\tau_{j}$ be the tube of length $C_{6}$ and radius $C_{6} \delta^{-\varepsilon} \sigma$ concentric with $T_{\ell_{j}}$. Here $C_{6}$ is a large constant which is chosen as follows: let $C_{4}$ be as in Lemma 3.4 and make $C_{6}$ large enough that if $d\left(\ell, \ell_{j}\right) \leq\left(C_{4}+2\right) \delta^{-\varepsilon} \sigma$ and $T_{m}$ intersects $T_{\ell}$ at angle less or equal than $\delta^{-\varepsilon} \sigma$ then $T_{m}$ is contained in $\tau_{j}$. It is easy to see that this is legitimate.

We will define subsets $F_{k} \subset E$ by a recursive construction. The logic here is similar to $\left[1\right.$, p. 154]. The $F_{k}$ will have the following properties: 
1) Each $F_{k}$ is assigned to a unique $\tau_{j}, j=j(k)$.

2) The $F_{k}$ assigned to a given $\tau_{j}$ are disjoint and are contained in

$$
\bigcup_{\ell \in \mathcal{B} \cap D\left(\ell_{j},\left(C_{4}+2\right) \delta^{-\varepsilon} \sigma\right)} Y_{\ell}^{0}
$$

(In particular, this implies they are contained in $\tau_{j}$, by choice of $C_{6}$ ).

3) Each $F_{k}$ is contained in a $C\left(\delta^{-2 \varepsilon} \phi_{k} \sigma+\delta\right)$-slab for a certain $\phi_{k} \leq \pi / 2$, and satisfies

$$
\left|F_{k}\right| \geq \delta^{C \varepsilon} N m^{-1 / 2} \lambda^{7 / 2} \delta \sqrt{\sigma} \sqrt{\phi_{k} \sigma+\delta} .
$$

4) $\sigma \sum_{k}\left(\phi_{k} \sigma+\delta\right) \geq \delta^{C \varepsilon} \lambda|\Omega|$.

To start the recursion, let $F_{0}=\varnothing$ and assign it to some arbitrary $\tau_{j}$. If $F_{i}$ has been defined for $i \leq k-1$, then for each tube in $\mathcal{B}$, we let

$$
Y_{\ell}=Y_{\ell}^{0} \backslash \cup\left\{F_{i}: i<k, F_{i} \text { assigned to } \tau_{j}\right.
$$

$$
\text { for some } \left.j \text { with } \ell \in D\left(\ell_{j},\left(C_{4}+2\right) \delta^{-\varepsilon} \sigma\right)\right\} \text {. }
$$

We throw out all $\ell \in \mathcal{B}$ such that $\left|Y_{\ell}\right| \leq\left|Y_{\ell}^{0}\right| / 2$. If half the lines in $\mathcal{B}$ are thrown out, we stop the induction. Otherwise, we let $\mathcal{B}_{k}$ be the remaining lines and note that the family $\mathcal{B}_{k}$ and the sets $Y_{\ell}$ satisfy the hypotheses of Lemma 3.4, since

$$
\left|Y_{\ell}\right| \geq \frac{1}{2}\left|Y_{\ell}^{0}\right| \geq\left(\log \frac{1}{\delta}\right)^{-3} \lambda\left|T_{\ell}\right|
$$

It follows that for some $\ell \in \mathcal{B}_{k}$ there is a set $F_{k} \subset \cup\left\{Y_{m}: m \in\right.$ $\left.\mathcal{B}_{k} \cap D\left(\ell, C_{4} \delta^{-\varepsilon} \sigma\right)\right\}$ and with property 3$)$. We choose $j$ so that $\ell \in$ $D\left(\ell_{j}, 2 \delta^{-\varepsilon} \sigma\right)$ and assign $F_{k}$ to this $\tau_{j}$. Then clearly $F_{k}$ is contained in $\cup\left\{Y_{m}: m \in D\left(\ell_{j},\left(C_{4}+2\right) \delta^{-\varepsilon} \sigma\right)\right\}$. It follows using (43) that $F_{k}$ is disjoint from $F_{i}$ if $i<k$ and $F_{i}$ is also assigned to $\tau_{j}$. This gives property 2 ).

It remains only to observe that when the induction stops property 4) will hold. This follows from the corollary to Lemma 3.5. Namely, if the induction stops at stage $k$ then at stage $k$ we have a subset $\mathcal{C} \subset \mathcal{B}$ of "thrown out" lines, with

$$
|\mathcal{C}| \geq \frac{1}{2}|\mathcal{B}| \geq \frac{1}{2}\left(\log \frac{1}{\delta}\right)^{-2}|\mathcal{A}|
$$


and therefore also $\mathcal{E}_{\delta}\left(\mathcal{C}^{*}\right) \gtrsim \delta^{2 \varepsilon}|\Omega|$ by (42). If $\ell \in \mathcal{C}$, then we let

$$
\begin{aligned}
E(\ell)=\cup\left\{F_{i}: i<k,\right. & F_{i} \text { assigned to } \tau_{j} \\
& \text { for some } \left.j \text { with } \ell \in D\left(\ell_{j},\left(C_{4}+2\right) \delta^{-\varepsilon} \sigma\right)\right\} .
\end{aligned}
$$

Since $\ell$ is thrown out we have

$$
\left|T_{\ell} \cap E(\ell)\right| \geq \frac{1}{2}\left(\log \frac{1}{\delta}\right)^{-2} \lambda\left|T_{\ell}\right| .
$$

We say that the $F_{i}$ in (44) are used in forming $E(\ell)$. If $C_{7}$ is a suitable constant then each set $F_{i}$ is contained in a $C_{7}\left(\delta^{-2 \varepsilon} \phi_{i} \sigma+\delta\right)$-slab, and if $\ell$ and $m$ are lines with dist $\left(\ell^{*}, m^{*}\right) \geq C_{7} \delta^{-\varepsilon} \sigma$, then no $\ell_{j}$ can be within $\left(C_{4}+2\right) \delta^{-\varepsilon} \sigma$ of both $\ell$ and $m$, so no $F_{i}$ is used in forming both $E(\ell)$ and $E(m)$. This gives the property (39) (with $\sigma$ replaced by $C \delta^{-\varepsilon} \sigma$ ). Accordingly (40) with $\sigma$ replaced by $C \delta^{-\varepsilon} \sigma$ implies

$$
\delta^{-\varepsilon} \sigma \sum_{i<k}\left(\delta^{-2 \varepsilon} \phi_{i} \sigma+\delta\right) \gtrsim \delta^{2 \varepsilon} \lambda \delta^{2 \varepsilon}|\Omega|,
$$

which gives 4$)$.

Next, using properties 3) and 4) we have

$$
\begin{aligned}
\sum_{k}\left|F_{k}\right| & \geq \delta^{C \varepsilon} N m^{-1 / 2} \lambda^{7 / 2} \delta \sum_{k}\left(\sigma\left(\phi_{k} \sigma+\delta\right)\right)^{1 / 2} \\
& \geq \delta^{C \varepsilon} N m^{-1 / 2} \lambda^{7 / 2} \delta\left(\sum_{k} \sigma\left(\phi_{k} \sigma+\delta\right)\right)^{1 / 2} \\
& \geq \delta^{C \varepsilon} N m^{-1 / 2} \lambda^{7 / 2} \delta(\lambda|\Omega|)^{1 / 2} \\
& =\delta^{C \varepsilon} N m^{-1 / 2} \lambda^{4} \delta|\Omega|^{1 / 2}
\end{aligned}
$$

Let $E_{j}=\cup\left\{F_{k}: F_{k}\right.$ assigned to $\left.\tau_{j}\right\}$. Then

$$
\sum_{j}\left|E_{j}\right|=\sum_{k}\left|F_{k}\right|
$$

by the disjointness property 2). On the other hand, we have

$$
E_{j} \subset \bigcup_{\ell \in D\left(\ell_{j},\left(C_{4}+2\right) \delta^{-\varepsilon} \sigma\right)} Y_{\ell}^{0}
$$


and since the $\left\{\ell_{j}\right\}$ are $\delta^{-\varepsilon} \sigma$-separated this implies (see the proof of the claim at the end of the proof of Lemma 2.1) that no point is in more than $C_{\varepsilon} E_{j}$ 's. We conclude that

$$
|E| \gtrsim \sum_{j}\left|E_{j}\right| \gtrsim \delta^{C \varepsilon} N m^{-1 / 2} \lambda^{4} \delta|\Omega|^{1 / 2}
$$

As in the proof of Lemma 2.1 (see (26)), we also have

$$
|E| \geq(2 N)^{-1} \sum_{\ell \in \mathcal{B}}\left|Y_{\ell}^{0}\right| \gtrsim \delta^{\varepsilon} \frac{\lambda \mathcal{E}_{\delta}(\mathcal{A})}{N}
$$

hence

$$
|E| \geq \delta^{2 \varepsilon} \frac{m \lambda|\Omega|}{N}
$$

by (41). If we combine this with (45) we get

$$
\begin{aligned}
|E| & \gtrsim \delta^{C \varepsilon}\left(N m^{-1 / 2} \lambda^{4} \delta|\Omega|^{1 / 2}\right)^{1 / 2}\left(\frac{\lambda m|\Omega|}{N}\right)^{1 / 2} \\
& =\delta^{C \varepsilon} \lambda^{5 / 2} m^{1 / 4}|\Omega|^{3 / 4} \delta^{1 / 2}
\end{aligned}
$$

and the proof of Lemma 0 is complete.

\section{Proofs of the theorems.}

Proof of Theorem 2. This is essentially the same as [7, Section 3]. The argument may appear simpler here however due to our attempt at abstraction in [7].

The idea is to induct downward on $\delta$. There is a technical point which must be dealt with first. Namely, in the preceding sections it was convenient to assume that $E$ was contained in the unit ball but this is now inconvenient, since we will want to use a rescaling argument. We take care of this issue in the next lemma.

Lemma 4.1. Assume that Theorem 2 is true for a certain value of $\delta$. Then the following variant is also true for the same value of $\delta$. Here the constants $C$ and $C_{\varepsilon}$ are the same as in (4) and $\beta$ is a numerical constant. 
Let $\Omega$ be a subset of $S^{2} \backslash \pm 1$, let $E$ be a subset of $\mathbb{R}^{3}$, and $\lambda>0$. Assume that for each $e \in \Omega$ there are $m \delta$-separated lines $\left\{\ell_{j}\right\}_{j=1}^{m}$, and points $\left\{a_{j}\right\}_{j=1}^{m}$ with $a_{j} \in \ell_{j}$, such that $\left|T_{\ell_{j}}\left(a_{j}\right) \cap E\right| \geq \lambda\left|T_{\ell_{j}}\left(a_{j}\right)\right|$. Then

$$
|E| \geq \beta C_{\varepsilon}^{-1}\left(\log \frac{1}{\delta}\right)^{-1} \delta^{C \varepsilon} \lambda^{5 / 2} m^{1 / 4}|\Omega|^{3 / 4} \delta^{1 / 2} .
$$

Proof. Let $\kappa$ and $\alpha$ be small constants chosen in that order. Subdivide $\mathbb{R}^{3}$ in cubes, $\mathbb{R}^{3}=\cup_{j \in \mathbb{Z}^{3}} Q_{j}$ where $Q_{j}$ is the cube centered at $\kappa j$ with sidelength $\kappa$. Denote $E_{j}=\left(Q_{j} \cap E\right)-\kappa j$, i.e. $E_{j}$ is the part of $E$ contained in $Q_{j}$, translated to the origin. Then $E_{j}$ is contained in the unit ball, and since any tube $T_{\ell}(a)$ intersects only a bounded number of cubes $Q_{j}$, one has the following: let $m_{j}(e)$ be the maximum possible cardinality for a $\delta$-separated set of lines $\ell$ in the $e$ direction such that $\left|T_{\ell} \cap E_{j}\right| \geq \alpha \lambda\left|T_{\ell}\right|$. Then $\sum_{j} m_{j}(e) \geq m$ for all $e \in \Omega$.

Hence also

$$
\sum_{j} \int_{\Omega} m_{j}(e) d e \geq m|\Omega|
$$

Note that $m_{j}(e) \lesssim \delta^{-2}$ for any $j$ and $e$. Accordingly there are numbers $\left\{\mu_{j}\right\}$ such that

$$
\int_{\left\{e \in \Omega: \mu_{j} \leq m_{j}(e) \leq 2 \mu_{j}\right\}} m_{j}(e) d e \gtrsim\left(\log \frac{1}{\delta}\right)^{-1} \int_{\Omega} m_{j}(e) d e
$$

and therefore

$$
\sum_{j} \mu_{j}\left|\Omega_{j}\right| \gtrsim \frac{m|\Omega|}{\log \frac{1}{\delta}}
$$

where $\Omega_{j}=\left\{e \in \Omega: \mu_{j} \leq m_{j}(e) \leq 2 \mu_{j}\right\}$. Because of the hypothesis that Theorem 2 is true with the given $\delta$, we then get

$$
|E|=\sum_{j}\left|E_{j}\right| \gtrsim C_{\varepsilon} \delta^{C \varepsilon} \lambda^{5 / 2} \delta^{1 / 2} \sum_{j} \mu_{j}^{1 / 4}\left|\Omega_{j}\right|^{3 / 4}
$$

where the implicit constant is purely numerical. On the other hand, clearly $\mu_{j} \leq m$ and $\left|\Omega_{j}\right| \leq|\Omega|$ for any $j$. Accordingly

$$
\sum_{j} \mu_{j}^{1 / 4}\left|\Omega_{j}\right|^{3 / 4} \geq \sum_{j} \frac{\mu_{j}\left|\Omega_{j}\right|}{m^{3 / 4}|\Omega|^{1 / 4}} \gtrsim \frac{m|\Omega|}{m^{3 / 4}|\Omega|^{1 / 4} \log \frac{1}{\delta}}=\frac{m^{1 / 4}|\Omega|^{3 / 4}}{\log \frac{1}{\delta}}
$$


If we substitute this into (46) we get the result.

Proof of TheOrem 2. As has already been mentioned the proof is by induction on $\delta$. By Lemma 2 we can choose $C$ and $A_{\varepsilon}$ so that if (3) and (5) hold then

$$
|E| \geq A_{\varepsilon}^{-1} \delta^{C \varepsilon} \lambda^{5 / 2} m^{1 / 4}|\Omega|^{3 / 4} \delta^{1 / 2} .
$$

Next we choose $\delta_{0}$ small enough that if $\delta<\delta_{0}$ then

$$
2^{-7 / 2} \beta\left(\log \frac{1}{\delta}\right)^{-26} \delta^{C \varepsilon(1-\varepsilon)}>\delta^{C \varepsilon} .
$$

Theorem 2 is trivial when $\delta \geq \delta_{0}$ provided $C_{\varepsilon}$ is large enough, so we can define a constant $C_{\varepsilon}$ by the following requirements:

- Theorem 2 is true with the given constant $C_{\varepsilon}$ provided $\delta \geq \delta_{0}$.

- $C_{\varepsilon} \geq 2 A_{\varepsilon}$.

Fix $\delta<\delta_{0}$ and assume that Theorem 2 has been proved with this value of $C_{\varepsilon}$ for parameters $\bar{\delta}, \bar{\delta} \geq \delta^{1-\varepsilon}$. Then under the assumptions of Theorem $2_{\delta}$, one of the following must happen:

1) There is a subset $\tilde{\Omega} \subset \Omega$ with measure greater or equal than $|\Omega| / 2$, such that if $e \in \tilde{\Omega}$ then there are $m / 2 \delta$-separated lines $\ell$ with direction $e$ such that (3) and (5) hold.

2) There is a subset $\tilde{\Omega} \subset \Omega$ with measure greater or equal than $|\Omega| / 2$, such that if $e \in \tilde{\Omega}$ then there are $m / 2 \delta$-separated lines $\ell$ with direction $e$ such that (3) holds and (5) fails.

In case 1 ), we simply apply Lemma 0 with $\Omega$ and $m$ replaced by $\tilde{\Omega}$ and $m / 2$ (more precisely, we use the second requirement on $C_{\varepsilon}$ ), obtaining the estimate

$|E| \geq A_{\varepsilon}^{-1} \delta^{C \varepsilon} \lambda^{5 / 2}\left(\frac{m}{2}\right)^{1 / 4}\left(\frac{|\Omega|}{2}\right)^{3 / 4} \delta^{1 / 2} \geq C_{\varepsilon}^{-1} \delta^{C \varepsilon} \lambda^{5 / 2} m^{1 / 4}|\Omega|^{3 / 4} \delta^{1 / 2}$,

which is the necessary inequality (4).

In case 2 ), we let $\bar{E}$ be $E$ dilated by a factor $\delta^{-\varepsilon}$. Fix $e \in \tilde{\Omega}$ and one of the $m / 2$ tubes in (2). Because of the hypothesis that (5) fails, there is a subtube of length $\delta^{\varepsilon}$ which intersects $E$ in measure greater or equal than

$$
\frac{1}{2} \lambda\left(\log \frac{1}{\delta}\right)^{-10}\left|T_{\ell}\right| .
$$


The dilation of this tube is a tube $\bar{T}_{\ell}$ of length 1 and radius $\bar{\delta} \stackrel{\text { def }}{=} \delta^{1-\varepsilon}$ which intersects $\bar{E}$ in measure greater or equal than

$$
\frac{1}{2} \lambda\left(\log \frac{1}{\delta}\right)^{-10} \delta^{\varepsilon}\left|\bar{T}_{\ell}\right|
$$

Thus (after dilation) for each $e \in \tilde{\Omega}$ we have $m / 2 \delta^{1-\varepsilon}$-separated $\ell$ 's generating such $\bar{T}_{\ell}$ 's. By the inductive hypothesis and Lemma 4.1 we have

$$
\begin{aligned}
\delta^{-3 \varepsilon}|E|=|\bar{E}| \geq & \beta C_{\varepsilon}^{-1}\left(\log \frac{1}{\delta}\right)^{-1} \bar{\delta}^{C \varepsilon} \\
& \cdot\left(\frac{1}{2} \delta^{-\varepsilon}\left(\log \frac{1}{\delta}\right)^{-10} \lambda\right)^{5 / 2}\left(\frac{m}{2}\right)^{1 / 4}\left(\frac{|\Omega|}{2}\right)^{3 / 4} \bar{\delta}^{1 / 2}
\end{aligned}
$$

or equivalently

$$
\begin{aligned}
|E| & \geq 2^{-7 / 2} \beta C_{\varepsilon}^{-1}\left(\log \frac{1}{\delta}\right)^{-26} \delta^{C \varepsilon(1-\varepsilon)} \lambda^{5 / 2} m^{1 / 4}|\Omega|^{3 / 4} \delta^{1 / 2} \\
& \geq C_{\varepsilon}^{-1} \delta^{C \varepsilon} \lambda^{5 / 2} m^{1 / 4}|\Omega|^{3 / 4} \delta^{1 / 2}
\end{aligned}
$$

where the last line follows from (47). This finishes the proof of Theorem 2 .

Proof of Theorem 1. Fix $\delta$ and define

$$
X_{\delta} f(\ell)=\left|T_{\ell}\right|^{-1} \int_{T_{\ell}} f .
$$

The first step is to prove

$$
\left\|X_{\delta} f\right\|_{L_{e}^{q} L_{x}^{r}} \lesssim \delta^{-C \varepsilon}\|f\|_{p}
$$

when $f$ is supported in the unit disc, with $p, q, r$ as in Theorem 1 .

A well-known argument (in this case it can be carried out by interpolation with $L^{\infty}$ and then with the result of [3]) shows that a bound like (48) which is insensitive to $\delta^{-\varepsilon}$ factors need only be proved for characteristic functions. So fix a set $E$, let $f=\chi_{E}$, and define $N=\left\|X_{\delta} f\right\|_{L_{e}^{q} L_{x}^{r}}$. We claim that for some $M$ there is a set $\Omega \subset S^{2}$ with

$$
|\Omega| \gtrsim\left(\log \frac{1}{\delta}\right)^{-1}\left(\frac{N}{M}\right)^{q}
$$


such that $e \in \Omega$ implies

$$
\left\|X_{\delta} f(e, \cdot)\right\|_{L_{x}^{r}} \geq M
$$

To see this, note that $X_{\delta} f$ is roughly constant on discs of radius $\delta$, in the sense (say) that if $X_{\delta} f\left(\ell_{0}\right)=\rho$, then $X_{\delta} f(\ell) \gtrsim \rho$ on a subset of $D\left(\ell_{0}, \delta\right)$ with measure $\gtrsim \delta^{4}$. Hence also

$$
\int_{D\left(e_{0}, \delta\right)}\left\|X_{\delta} f(e, \cdot)\right\|_{L_{x}^{r}}^{q} d e \geq \delta^{2}\left\|X_{\delta} f\left(e_{0}, \cdot\right)\right\|_{L_{x}^{r}}^{q},
$$

for any $e_{0} \in S^{2}$, so that

$$
\sup _{e}\left\|X_{\delta} f(e, \cdot)\right\|_{L_{x}^{r}}^{q} \leq \delta^{-2} N^{q}
$$

So if we let

$$
J=\left\{e \in S^{2}: C^{-1} N^{q} \leq\left\|X_{\delta} f(e, \cdot)\right\|_{L_{x}^{r}}^{q} \leq C \delta^{-2} N^{q}\right\},
$$

then

$$
\int_{J}\left\|X_{\delta} f(e, \cdot)\right\|_{L_{x}^{r}}^{q} d e \gtrsim N^{q}
$$

Split the integral over $J$ into the regions $\Omega_{j}$ where $\left\|X_{\delta} f(e, \cdot)\right\|_{L_{x}^{r}}^{q} \in$ $\left(2^{j}, 2^{j+1}\right)$ and note that there are $\lesssim \log (1 / \delta)$ relevant values of $j$. Hence the claim holds for some $M=2^{j / q}$ and $\Omega=\Omega_{j}$.

Next, by a similar argument there are $m$ and $\lambda$ with $m \delta^{2} \lambda^{r} \gtrsim$ $\delta^{\varepsilon} M^{r}$ and $\tilde{\Omega} \subset \Omega$ with $|\tilde{\Omega}| \gtrsim \delta^{\varepsilon}|\Omega|$ such that if $e \in \tilde{\Omega}$ then $X_{\delta} f(e, x) \geq \lambda$ for a set of $x$ of measure at least $m \delta^{2}$. Equivalently, if $e \in \tilde{\Omega}$ then there are $m \delta$-separated lines $\ell$ with direction $e$ and with $\left|E \cap T_{\ell}\right| \geq \lambda\left|T_{\ell}\right|$. We conclude by Theorem 2 that

$$
\begin{aligned}
|E| & \gtrsim \delta^{C \varepsilon} \lambda^{5 / 2}\left(m \delta^{2}\right)^{1 / 4}|\Omega|^{3 / 4} \\
& =\delta^{C \varepsilon}\left(m \delta^{2} \lambda^{r}\right)^{1 / 4}|\Omega|^{3 / 4} \\
& \gtrsim \delta^{C \varepsilon} M^{r / 4}\left(\frac{N}{M}\right)^{3 q / 4} \\
& =\delta^{C \varepsilon} N^{5 / 2}
\end{aligned}
$$

so we have proved (48). To finish the proof of Theorem 1 we have to trade the $\delta^{\varepsilon}$ factor for $\varepsilon$ derivatives. This is a standard argument. We 
choose a $C_{0}^{\infty}$ function $\psi$ with $\operatorname{supp} \psi \subset D(0,1 / 1000)$, and a Schwarz function $\rho$ such that $\hat{\rho}$ has compact support not containing the origin, such that

$$
\hat{\eta} \stackrel{\text { def }}{=} 1-\sum_{j \geq 0} \widehat{\psi_{j}} \widehat{\rho_{j}} \in C_{0}^{\infty},
$$

where $\psi_{j}(x)=2^{3 j} \psi\left(2^{j} x\right), \rho_{j}(x)=2^{3 j} \rho\left(2^{j} x\right)$. It is easy to see that this is possible. Here are details since we don't have a reference at hand: start with a $C_{0}^{\infty}$ function $\phi$ supported in $D(0,1 / 2000)$ with $\hat{\phi}(0) \neq 0$. By multiplying $\phi$ by a character we can insure that $\hat{\phi}$ does not vanish identically on any sphere centered at 0 . Let $\phi_{2}=\tilde{\phi} * \phi$ where $\tilde{\phi}(x)=$ $\overline{\phi(-x)}$. Then $\widehat{\phi_{2}}=|\hat{\phi}|^{2}$. Let $\psi=\sum_{i} \phi_{2} \circ T_{i}$ where $\left\{T_{i}\right\}$ is an appropriate finite set of rotations. By a compactness argument we can arrange that $\hat{\psi}$ be nonzero on $D(0,2)$ say. Next choose a partition of unity of the form $\left\{\chi_{j}\right\}_{j=-\infty}^{\infty}$ where $\chi_{j}(\xi)=\chi_{0}\left(2^{-j} \xi\right)$. Define $\rho_{j}$ via $\hat{\rho}_{j}=\chi_{j} / \hat{\psi}_{j}$, $j \geq 0$.

Furthermore, let $\gamma$ be a $C_{0}^{\infty}$ cutoff function equal to 1 on $D(0,1 / 100)$ and supported in $D(0,1 / 10)$.

In proving Theorem 1 we can suppose that $f$ is supported in $D(0,1 / 1000)$ and $\|f\|_{p, \varepsilon}=1$. Then in the first place,

$$
\sum_{j} 2^{\eta j}\left\|\rho_{j} * f\right\|_{p} \leq C_{\varepsilon}
$$

if (say) $\eta<\varepsilon / 2$; this follows easily using the definition of the Sobolev space and the support property of $\hat{\rho}$. In the second place, using the support properties we have

$$
f=\eta * f+\sum_{j} \psi_{j} *\left(\gamma \cdot\left(\rho_{j} * f\right)\right),
$$

on $\operatorname{supp} f$, and therefore

$$
|X f| \leq X(|\eta * f|)+\sum_{j} X\left(\left|\psi_{j} *\left(\gamma \cdot\left(\rho_{j} * f\right)\right)\right|\right) .
$$

The first term is less or equal than $C$ pointwise. For the remaining terms, we use that $X\left(\left|\psi_{j} * g\right|\right)\left|\leq C X_{\delta_{j}}\right| g \mid$ pointwise if $\operatorname{supp} g \subset$ $D(0,1 / 10)$, where $\delta_{j}=2^{-j}$. This is clear from the definitions and the compact support of $\psi$. Applying (48) with $\varepsilon$ in (48) taken to be small compared with the current $\varepsilon$, we obtain

$$
\left\|X\left(\left|\psi_{j} *\left(\gamma \cdot\left(\rho_{j} * f\right)\right)\right|\right)\right\|_{L_{e}^{q} L_{x}^{r}} \lesssim\left\|X_{\delta_{j}}\left(\left|\gamma \cdot \rho_{j} * f\right|\right)\right\|_{L_{e}^{q} L_{x}^{r}} \leq 2^{\eta j}\left\|\gamma \cdot \rho_{j} * f\right\|_{p},
$$


with $\eta<\varepsilon / 2$. Theorem 1 now follows by (49).

Concluding Remarks. 1) The following is an easy corollary of Theorem 1 or 2 :

Let $E$ be a Borel subset of $\mathbb{R}^{3}$ and assume that for each $e \in S^{2}$, there is a Borel set $L_{e} \subset e^{\perp}$ with Hausdorff dimension at least $\beta$ such that for each $x \in L_{e}$, some segment of the line through $x$ in the $e$ direction is contained in $E$. Then the Hausdorff dimension of $E$ is at least $5 / 2+\beta / 4$.

Here $e^{\perp}$ is the orthogonal complement of $e$ in $\mathbb{R}^{3}$. We omit the proof. It follows a standard pattern originating (to the author's knowledge) in [1].

2) We make a few remarks about the open question of whether or not the exponent $5 / 2$ in the Kakeya problem can be improved. For example, let $E$ be a compact set containing a unit line segment $\ell_{e}$ in every direction $e$. Is its Minkowski dimension (i.e.

$$
3-\limsup _{\delta \rightarrow 0} \frac{\log \left|E^{\delta}\right|}{\log \delta}
$$

$E^{\delta}=\delta$-neighborhood of $E$ ) strictly greater than $5 / 2$ ? Theorem 2 shows that the enemy is the case where the lines "stick together" in the sense that $d\left(\ell_{e}, \ell_{e^{\prime}}\right) \approx\left|e-e^{\prime}\right|$ up to $\delta^{-\varepsilon}$ factors. The reason is that if this condition fails in too dramatic a way, then the sets $E^{\delta}$ will contain not just one but many $\delta$-tubes per direction and Theorem 2 will be applicable with a large value of $m$. For example, one can reduce in this way to the case where the following condition $(*)$ is satisfied:

$(*)$ For any $\varepsilon>0$ there is a sequence of $\delta$ going to 0 such that the set $\left(e, e^{\prime}\right) \in S^{2} \times S^{2}: d\left(\ell_{e}, \ell_{e^{\prime}}\right) \leq \delta$ has measure greater or equal than $\delta^{\varepsilon} \delta^{2}$.

At the opposite extreme, if the inequality $d\left(\ell_{e}, \ell_{e^{\prime}}\right) \approx\left|e-e^{\prime}\right|$ holds in the strict sense that

$$
d\left(\ell_{e}, \ell_{e^{\prime}}\right) \leq C\left|e-e^{\prime}\right|
$$

for all $e$ and $e^{\prime}$ then it is easy to show using Rademacher's theorem on almost everywhere differentiability of Lipschitz functions (e.g. [4]) that $E$ will have positive measure. 
We indicate the proof (in $\mathbb{R}^{n}$ ) assuming that for each $e, E$ contains a segment of $\ell_{e}$ with length 1 which intersects the plane $x_{n}=0$; only minor modifications are required to treat the general case. First let $U \subset \mathbb{R}^{n}$ be open and let $F: U \longrightarrow \mathbb{R}^{m}$ be any Lipschitz function. We claim that if $\varepsilon>0$ then there are a subset $Y \subset U$ with positive measure, and a linear map $T: \mathbb{R}^{n} \longrightarrow \mathbb{R}^{m}$ such that

$$
|F(x)-F(y)-T(x-y)| \leq \varepsilon|x-y|,
$$

for all $x \in Y, y \in Y$. Namely, let $D F(a)$ be the derivative of $F$ at $a$ given by Rademacher's theorem. By the Lusin and Egoroff theorems there is a positive measure subset $Y_{0}$ such that $D F(a)$ is continuous on $Y_{0}$ as a function of $a$, and furthermore the difference quotients

$$
\frac{|F(x)-F(a)-D F(a)(x-a)|}{|x-a|}
$$

converge to 0 as $x \longrightarrow a$ uniformly over $a \in Y_{0}$. Let $\delta$ be small enough and let $a$ be a point of density of $Y_{0}$. Let $Y=Y_{0} \cap D(a, \delta)$. Let $T=D F(a)$. Then for $x, y \in Y$, the properties of $Y_{0}$ imply

$$
\begin{aligned}
\mid F(x) & -F(y)-T(x-y) \mid \\
& \leq|F(x)-F(y)-D F(y)(x-y)|+|D F(y)(x-y)-T(x-y)| \\
& \leq \varepsilon|x-y|
\end{aligned}
$$

as claimed.

Now parametrize (an appropriate subset of) projective space via $e=(\xi, 1), \xi \in \mathbb{R}^{n-1}$ and define a family of maps $F_{t}$ from a suitable subset of $\mathbb{R}^{n-1}$ to $\mathbb{R}^{n-1}$ by letting $\left(F_{t}(\xi), t\right)$ be the intersection point between $\ell_{e}$ and the plane $x_{n}=t$. Note that $F_{t}(\xi)=F_{0}(\xi)+t \xi . F_{0}$ is Lipschitz, so we can choose $Y$ and a linear map $T$ so that $Y$ has positive measure and

$$
\left|F_{0}(\xi)-F_{0}(\eta)-T(\xi-\eta)\right|<\varepsilon|\xi-\eta|, \quad \xi, \eta \in Y
$$

where $\varepsilon$ is to be determined. We then have

$$
\left|F_{t}(\xi)-F_{t}(\eta)-(t I+T)(\xi-\eta)\right| \leq \varepsilon|\xi-\eta|,
$$

when $\xi, \eta \in Y$, where $I$ is the identity map. Hence $F_{t}$ is bilipschitz on $Y$ provided that $\varepsilon\left\|(t I+T)^{-1}\right\|<1$, which will be the case for all $t$ except 
a set of measure less or equal than $C \varepsilon^{1 /(n-1)}$. We are free to choose $\varepsilon$ small, so the result follows using the fact that a bilipshitz image of a set of positive measure has positive measure and then Fubini's theorem.

However, it appears difficult to replace the strict sense condition (50) with a similar condition (e.g. $(*))$ which is weak enough to be useful, even if one asks only for the weaker conclusion $\operatorname{dim}(E)>5 / 2$.

Acknowledgments. Wilhelm Schlag pointed out an inaccuracy in a preliminary version of the paper, and Terry Tao pointed out some obscurities in the exposition.

\section{References.}

[1] Bourgain, J., Besicovitch type maximal operators and applications to Fourier. Geometric and Functional Analysis 1 (1990), 147-187.

[2] Christ, M., Estimates for the $k$-plane transform. Indiana Univ. Math. J. 33 (1984), 891-910.

[3] Drury, S., $L^{p}$ estimates for the X-ray transform. Illinois J. Math. 27 (1983), 125-129.

[4] Evans, L. C., Partial Differential Equations. Amer. Math. Soc. 1998.

[5] Nathanson, M., Additive Number Theory: inverse problems and the geometry of sumsets. Springer-Verlag, 1996.

[6] Oberlin, D., Stein, E. M., Mapping properties of the Radon transform. Indiana Univ. Math. J. 31 (1982), 641-650.

[7] Wolff, T., An improved bound for Kakeya type maximal functions. Revista Mat. Iberoamericana 11 (1995), 651-674.

Recibido: 26 de mayo de 1.997

Thomas Wolff

Department of Mathematics

253-37 Caltech

Pasadena, CA 91125, USA

wolff@cco.caltech.edu 